\title{
ESTRUCTURAS ESPACIALES DESPLEGABLES CURVAS
}

\author{
(EXPANDABLE CURVED SPACE BAR STRUCTURES)
}

Félix Escrig, Prof. Titular de la ETSA de Sevilla

J. P. Valcárcel, Prof. Titular de la ETSA de La Coruña

\section{RESUMEN}

Las estructuras desplegables de barras basadas en aspas están siendo investigadas intensamente $y$ ofrecen muchas posibilidades de utilización en arquitectura. De ellas, las que toman formas que puedan cerrar recintos son las más atractivas y con más futuro. En el articulo se hace una sintesis de las disposiciones formales para obtener cubiertas cilíndricas y esféricas con estructuras que sean estables y compatibles, y se repasa una amplia gama de las tipologías con más posibilidades o que han sido objeto de estudio. Igualmente se hace una introducción al cálculo matricial de sus elementos y conjuntos con las nociones básicas para la confección de un programa especifico.

Brevemente se comenta también la aplicación del cálculo al estudio de algunos ejemplos concretos.

\section{SUMMARY}

Expandable Space Bas Structures are being intensively investigated and they offer great utilitiers in architecture. They can be adjusted to a range of shapes as varied as for any other type of articulated structures. Among them, those used to enclose spaces are the most attractive and credited for the future. In this paper some cylindrical and spherical design models are discussed and classified according to their generation pattern and their kynematic constraints, taking on account requirements to be fulltilled to make their geometry compatible with the stages of unfolding. An introduction to the method for structural analysis is also included, along with a number of examples.

\section{INTRODUCCION}

Estructuras desplegables de barras son conjuntos de piezas articuladas que pueden transportarse en paquetes compactos que no ocupan más espacio que el del propio haz de barras concentrado y que, por procedimientos manuales o mecánicos, pueden desplegarse en los lugares en donde se requiera su uso para crear recintos arquitectónicos, cubrir espacios deportivos 0 de espectáculos, montar instalaciones de uso militar o civil, generar superficies para captación de energía en tierra o en el espacio, formar depósitos o contenedores o constituir elementos auxiliares tales como escaleras, antenas, soportes publicitarios, apeos y auxiliares de construcción, puentes y viaductos, etc. sin más limitaciones que las propias de cualquier estructura rígida.

El proceso de desplegado puede ser más o menos complejo dependiendo del trabajo y la especialización que requiera y la cantidad de medios auxiliares necesarios.

Nosotros vamos a plantear el funcionamiento de unas estructuras cuyo proceso de apertura y cierre sea tan elemental que pueda realizarse en pocos minutos y con los propios dispositivos internos del sistema; es decir, estructuras que pueden tomar varias configuraciones en el espacio y bloquearse en varias de ellas, fundamentalmente en estado de máximo desarrollo o de mínimo volumen.

Las tipologías de estructuras que cumplen con estos requisitos y que tienen una bibliografía mínima y unas aplicaciones estudiadas son:

a) Tipo paraguas. Estructuras que se pliegan sobre un mástil mediante el deslizamiento de un punto de apoyo sobre él. Son estructuras adecuadas para luces pequeñas, no mayores de $5 \mathrm{~m}$ y tienen su utilidad como mobiliario urbano.

b) Tipo mecanismo con articulaciones bloqueables. Tal como se expresa genéricamente en la Fig. 1 en donde las articulaciones en el interior de las barras, una vez la estructura se ha abierto, se bloquean y la hacen rígida. Tienen el inconveniente de que el proceso de cierre es complicado y requiere soltar uno a uno todos los cierres, pero en cambio son estructuras muy rígidas y que se usan frecuentemente en instalaciones aeroespaciales. 


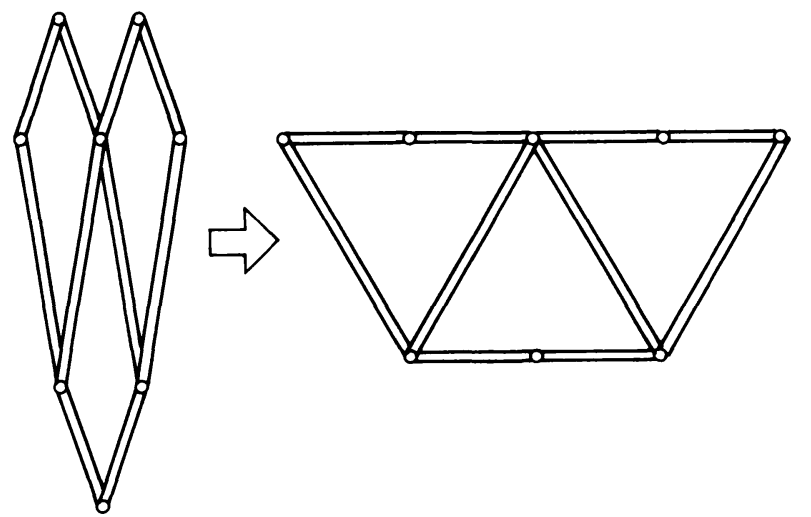

Fig. 1

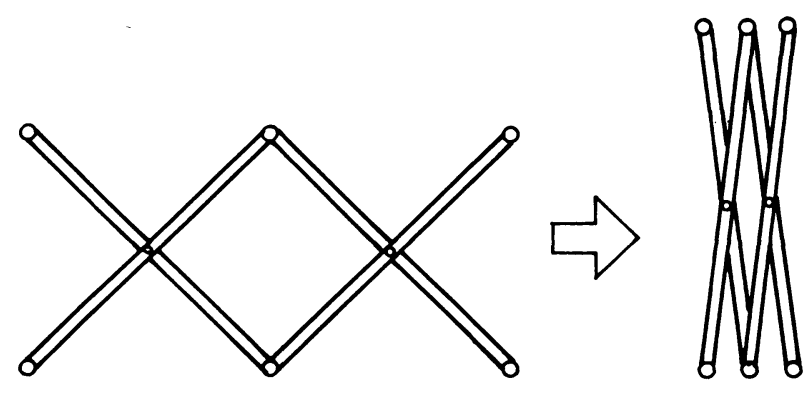

Fig. 2
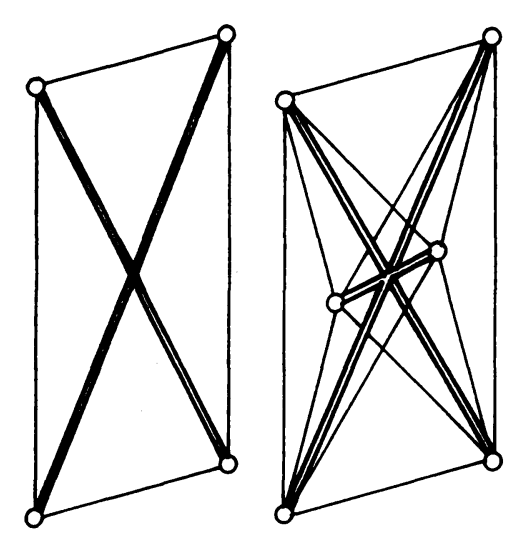

Fig. 3

c) Tipo aspa. Son las estructuras a que nos vamos a referir en el presente trabajo y se basan en las distintas posiciones que puede tomar un elemento como el de la Fig. 2. Estas estructuras están bien estudiadas en sus configuraciones elementales en las referencias 4,5 y 6 , y son mecanismos que se obtienen combinando espacialmente piezas como las de la Fig. 2 o como los módulos de la Fig. 3. El conjunto es móvil porque es inestable y puede plegarse y desplegarse sometido únicamente a las leyes de la compatibilidad geométrica de las barras. Para que nos sivan como estructura, estos conjuntos deben estabilizarse en alguna posición. Pero a diferencia de lo que ocurría en las estructuras de articula-
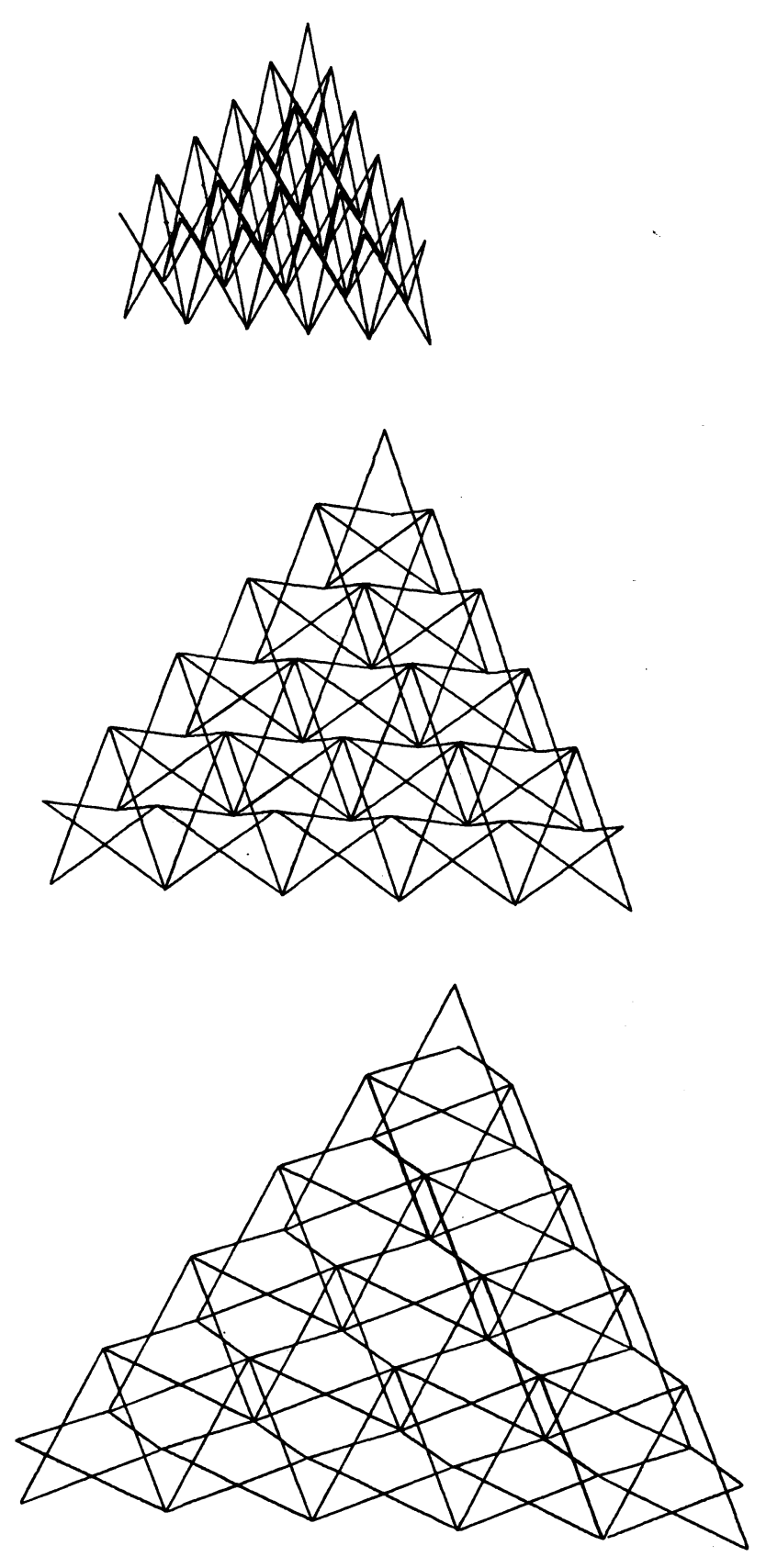

Fig. 4

ción bloqueable, basta con fijar dos nudos para que toda la estructura funcione. La Fig. 4 muestra un gráfico del desplegado de una de estas estructuras.

\section{COMPATIBILIDAD GEOMETRICA}

Las estructuras más sencillas que podemos construir con estos elementos son las planas, constituidas por barras de igual longitud y articulación en su punto medio. A partir de estas estructuras planas basadas en el aspa (Fig. 5a), podemos obtener mallas curvas situando excéntrico el punto de cruce, lo que da lugar a un crecimiento como el de la (Fig. 5b). La composición es- 
a

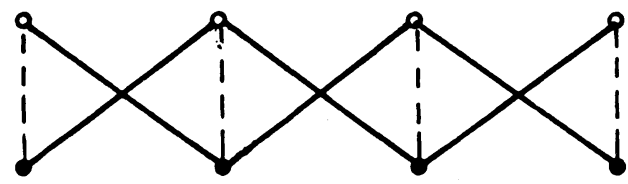

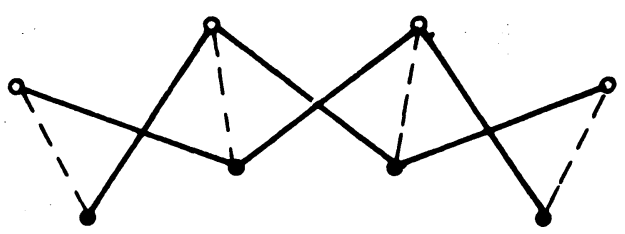

b

Fig. 5

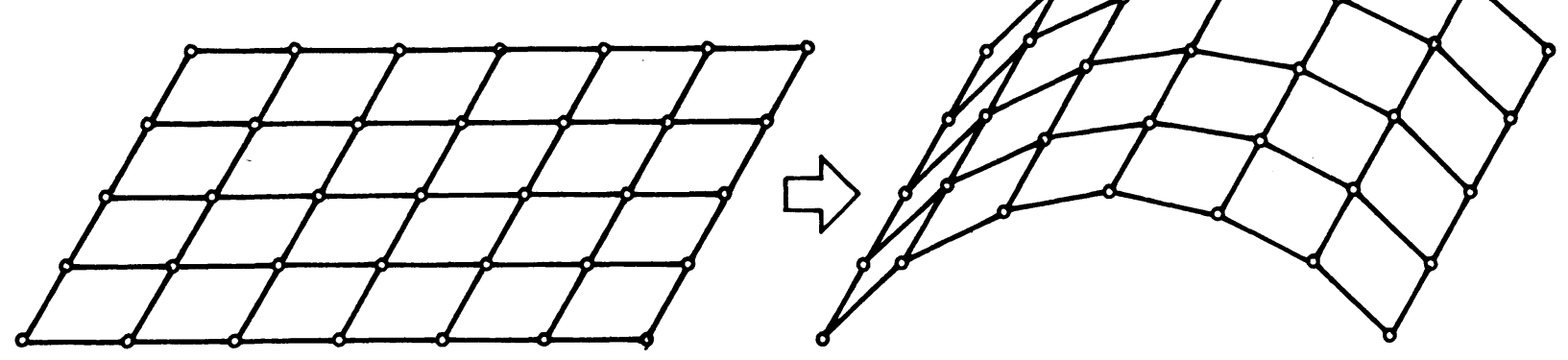

Fig. 6

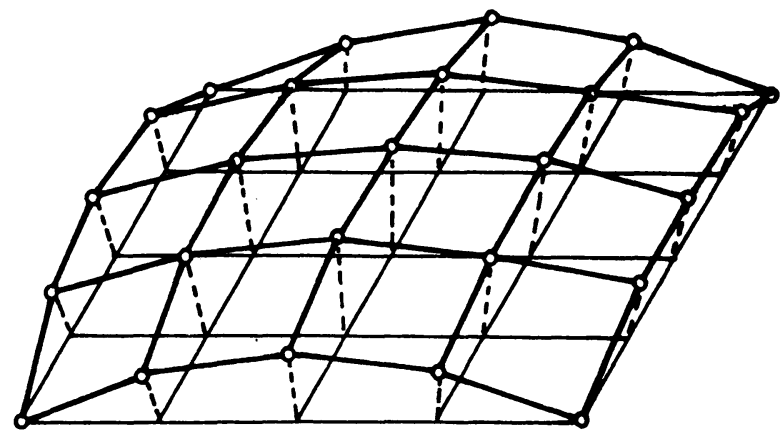

Fig. 7

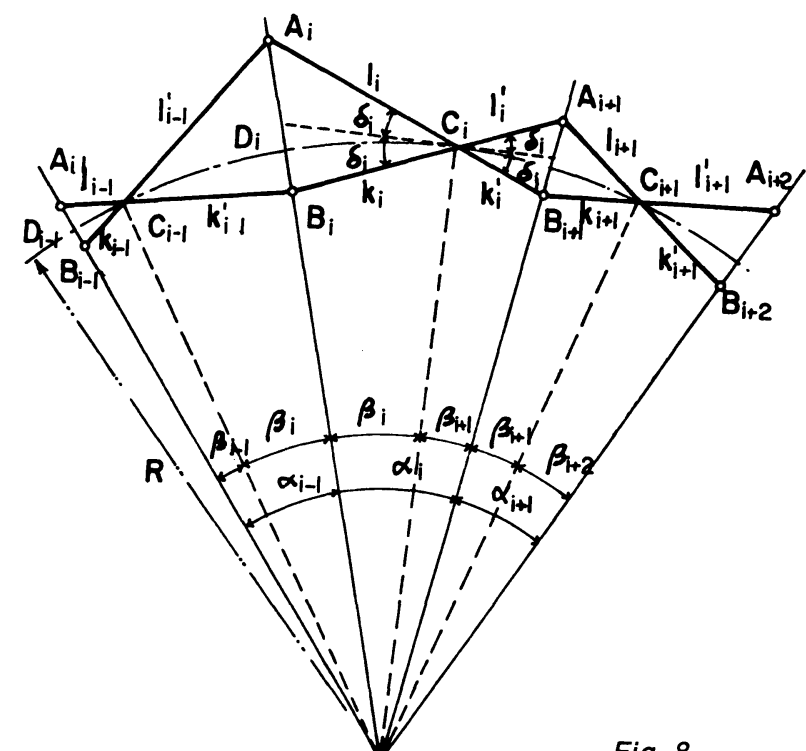

Fig. 8

C Consejo Superior de Investigaciones Científicas Licencia Creative Commons 3.0 España (by-nc) pacial de sistemas como éste o planos da lugar a la obtención de mallas complejas que siguen manteniendo la capacidad de plegado o desplegado. No obstante, las mallas curvas tienen algunas dificultades geométricas que requieren ser analizadas para obtener las configuraciones más simples que sean al mismo tiempo estables y compatibles. Las configuraciones que vamos a estudiar se basan todas en superficies de traslación o de revolución con directriz circular y se generan a partir de mallas planas que se curvan en el caso de superficies cilindricas (Fig. 6) o se proyectan en el caso de superficies esféricas (Fig. 7).

Para posibilitar la adaptación de una malla plegable a estas retículas curvas vamos a adoptar los siguientes criterios (Fig. 8):

a) La superficie generatriz (cilindro o esfera) contendrá todos los puntos "C" de cruce de las aspas.

b) Los nudos de la retícula lineal serán los " $D$ " y, tanto los nudos superiores como los inferiores de la malla plegable, estarán sobre los radios que pasan por "D".

c) Para que el plegado sea posible debe cumplirse:

$l_{\mathrm{i}-1}+\mathrm{K}_{\mathrm{i}-1}=l_{\mathrm{i}}+\mathrm{K}_{\mathrm{i}}$

Y, en el caso de directrices circulares, además:

$l_{\mathrm{i}-1}=l_{\mathrm{i}}$

$\mathrm{K}_{\mathrm{i}-1}=\mathrm{K}_{\mathrm{i}}$

http://informesdelaconstruccion.revistas.csic.es 
Esto es 10 mismo que plantear que:

$\delta_{i-1}=\delta_{i}=\delta_{i+1}=\ldots=\delta_{n}$

De este modo:

$$
\begin{aligned}
l_{\mathrm{i}}=l_{\mathrm{i}-1} & =\frac{\mathrm{R} \operatorname{sen} \beta_{\mathrm{i}}}{\cos \left(\delta+\beta_{\mathrm{i}}\right)} \\
\mathrm{k}_{\mathrm{i}}=\mathrm{k}_{\mathrm{i}-1} & =\frac{\mathrm{R} \operatorname{sen} \beta_{\mathrm{i}}}{\cos \left(\delta-\beta_{\mathrm{i}}\right)}
\end{aligned}
$$

Como el radio es variable en las distintas fases del desplegado, podemos tomar su valor en la posición final desplegada, en donde habrá que fijar también el valor " $\delta$ " que debe abrir el aspa.

Para que la estructura funcione $\delta_{i}>\beta_{i}$, pues de lo contrario la estructura se acodala y ya no es posible el plegado (Fig. 9).

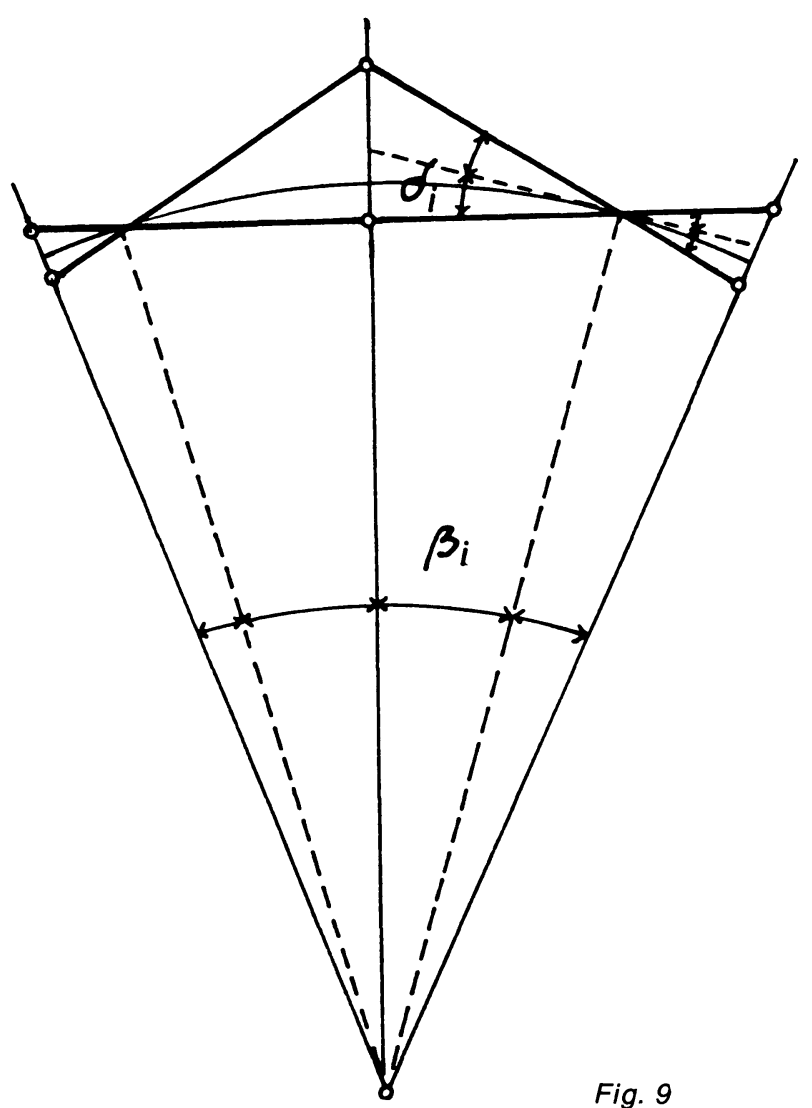

\section{MALLAS CILINDRICAS RECTANGULARES}

Si partimos de una malla cuadrada como la de la Fig. 10 , en la cual se combinan dos familias de vigas $A$ y $B$, curvando una de ellas, obtenemos la superficie cilíndrica de la Fig. 11.
Las mallas planas y curvas tienen las mismas propiedades estructurales. En este caso la estabilidad angular podría conseguirse diagonalizando algunos módulos mediante barras (Fig. 12). Pero estas barras son más largas que las restantes y, por tanto, impiden el plegado. La utilización de barras telescópicas, cables o, incluso la propia cubierta textil, podrian ser otras alternativas.

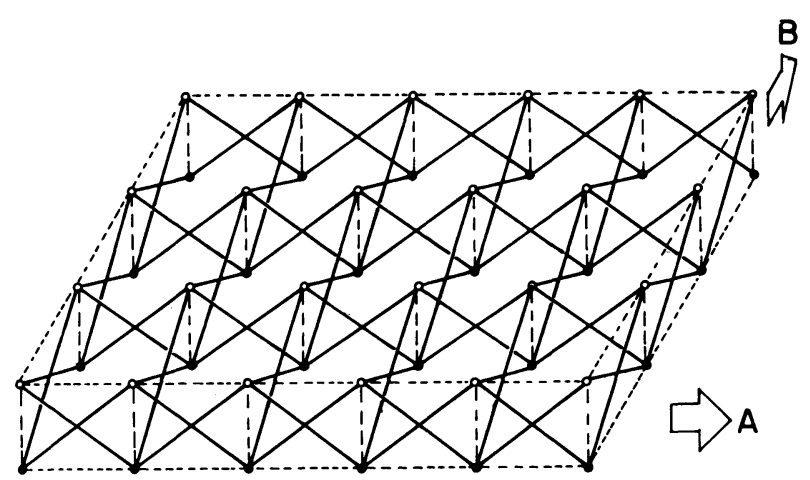

Fig. 10

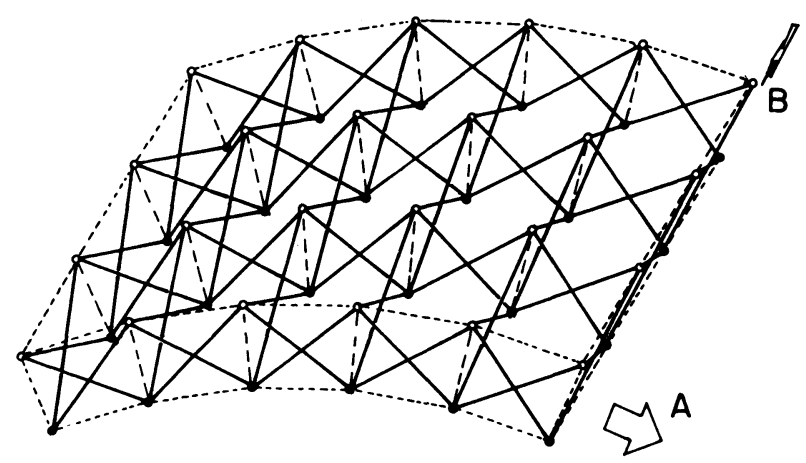

Fig. 11

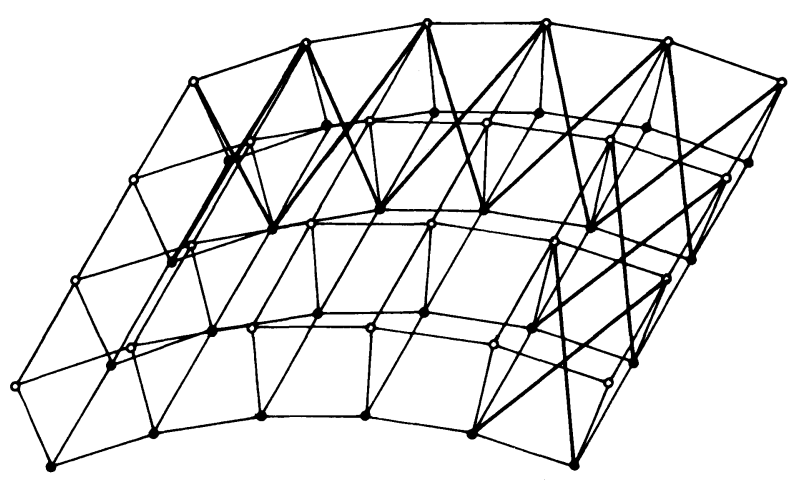

Fig. 12 


\section{MALLAS CILINDRICAS TRIANGULARES}

De un modo similar, si partimos de una malla triangular como la de la Fig. 13, con tres familias de vigas $A$, $B$ y $C$, podemos conseguir superficies cilindricas curvando dos o tres de ellas según se ve en las Figs. 14, $15,16$ y 17$)$.

La Fig. 15 representa una configuración en la cual las vigas de la familia $A$ se curvan en un plano mientras que las $B$ y $C$ se alabean. La Fig. 14 es una simplificación de la Fig. 15 en que se representan sólo los cuadriláteros circunscritos a las aspas por claridad de representación. En adelante todas las mallas tendrán esta representación alternativa que ayudará a comprenderlas.

La Fig. 17 representa una configuración en que las vigas tipo A permanecen planas, mientras que las tipo $B$ y $C$ se alabean. Este sistema es menos estable que el anterior. En los dos casos el alabeo introduce una dificultad adicional puesto que no es posible emplazar aspas en un cuadrilátero alabeado. Ambas diagonales del cuadrilátero se cruzan en el espacio y, por tanto, no tienen ningún punto intermedio de contacto. Las estructuras desplegables cilíndricas triangulares de este tipo son una combinación de aspas y barras aisladas (Fig. 18). Los análisis realizados en este tipo de estructuras confirman que siguen siendo estables si se inmovilizan los puntos de apoyo.

Otra posibilidad para resolver el problema es el uso de barras curvas que, en la posición de plegado, deben forzarse hacia la configuración recta, almacenando así una energía potencial que puede utilizarse para autodesplegar la estructura y fijarla en su posición final (Fig. 19).

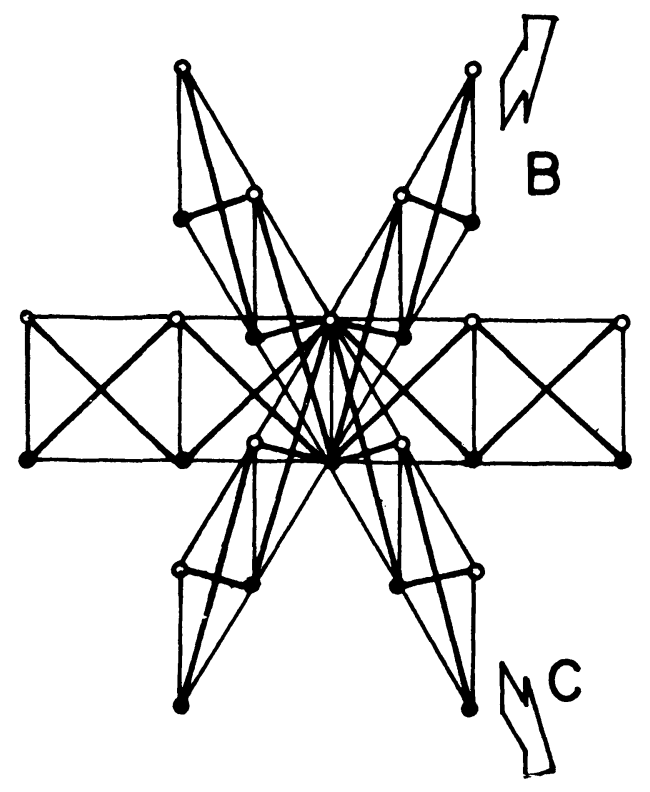

Fig. 13

(c) Consejo Superior de Investigaciones Científicas Licencia Creative Commons 3.0 España (by-nc)

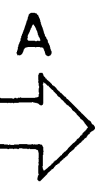

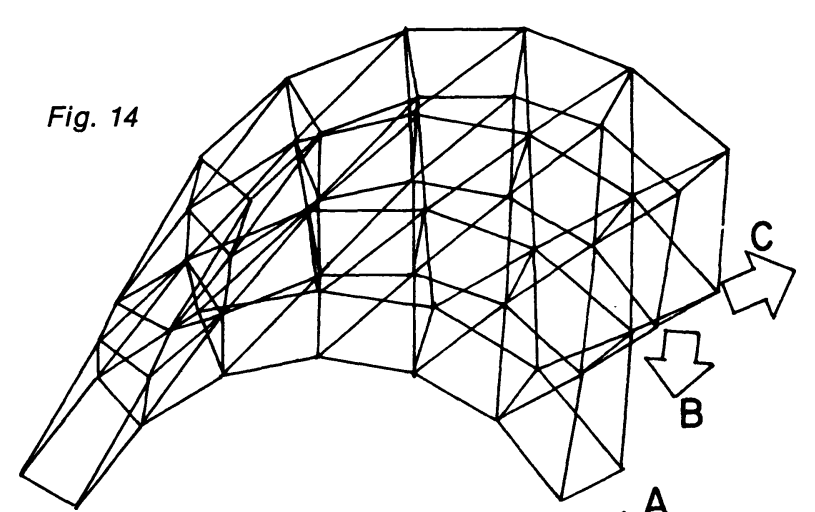

$\sum \jmath^{A}$
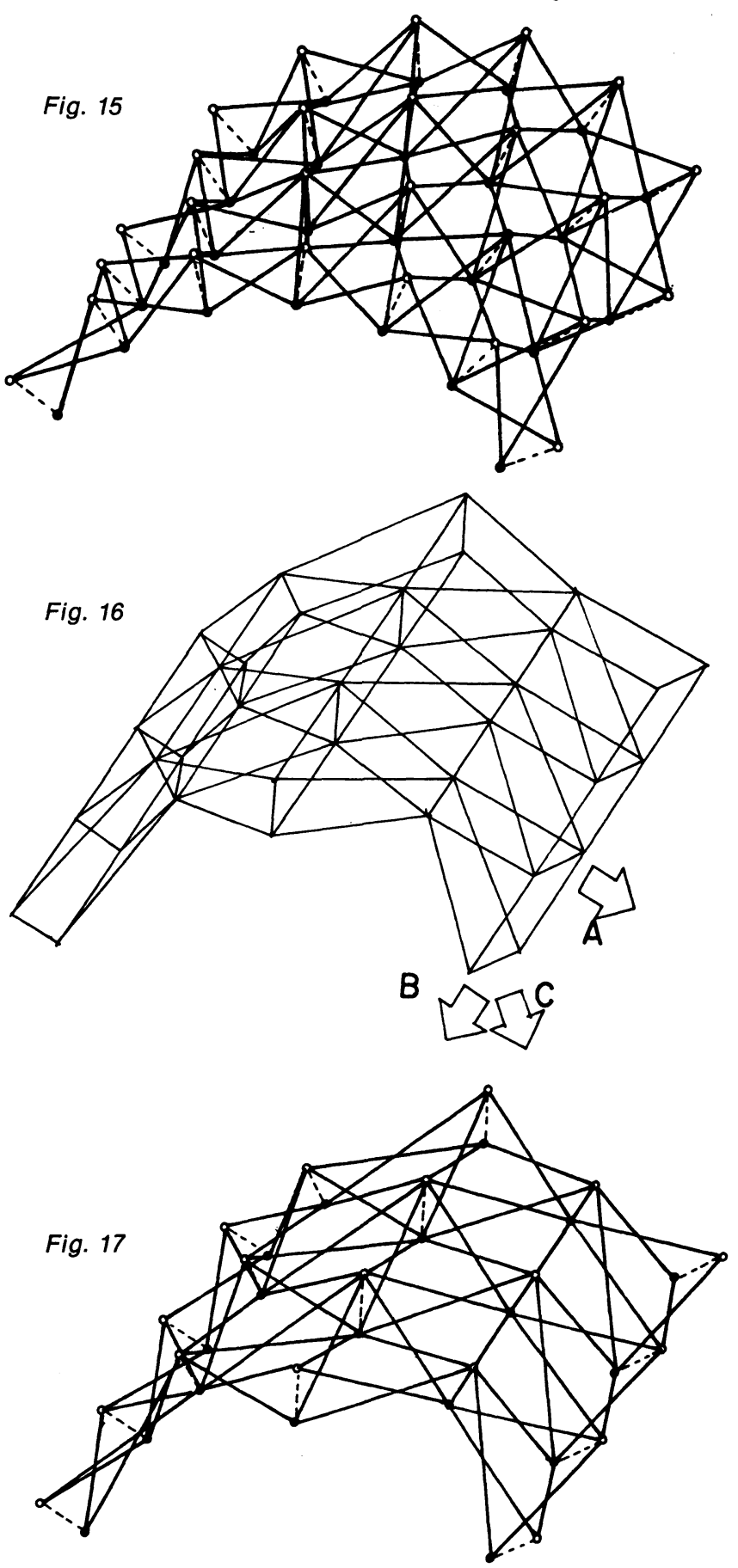

http://informesdelaconstruccion.revistas.csic.es 


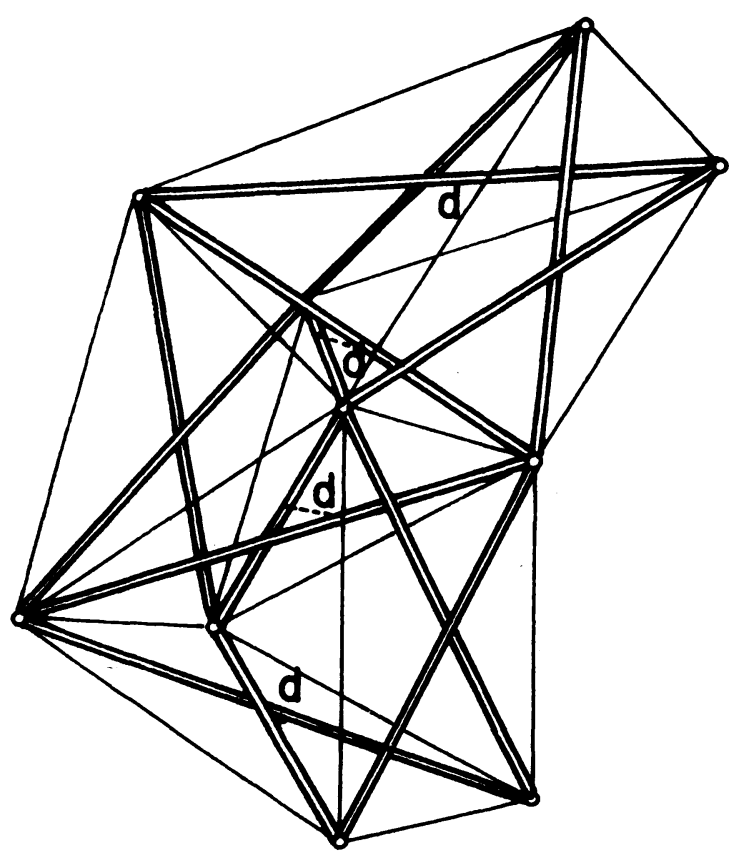

Fig. 18

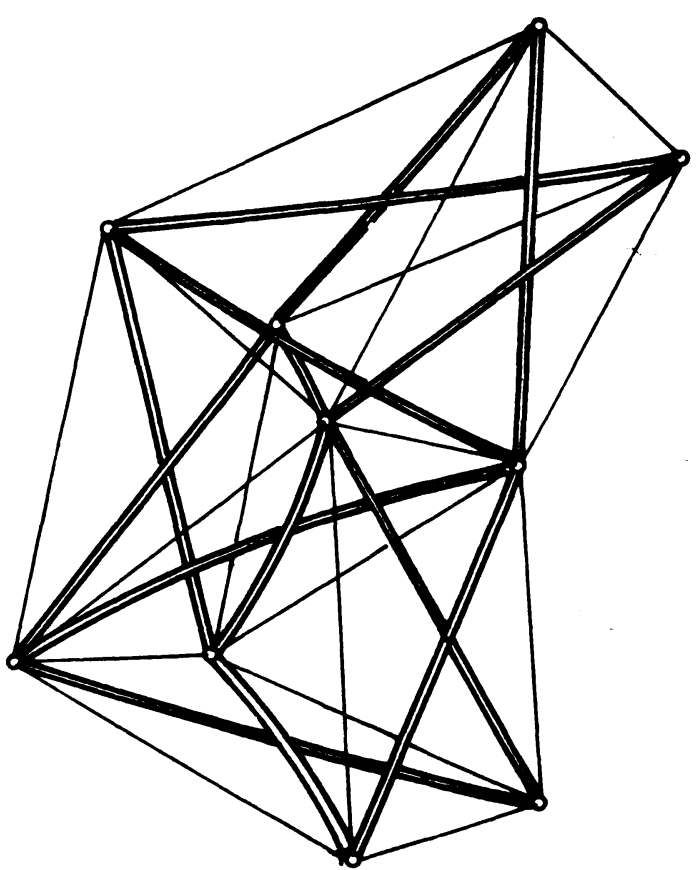

Fig. 19

curvatura. Si todas las aspas son iguales se obtiene una superficie esférica como la de la Fig. 21 en que se han ido trazando las distancias sobre la superficie esférica para obtener la malla correspondiente.
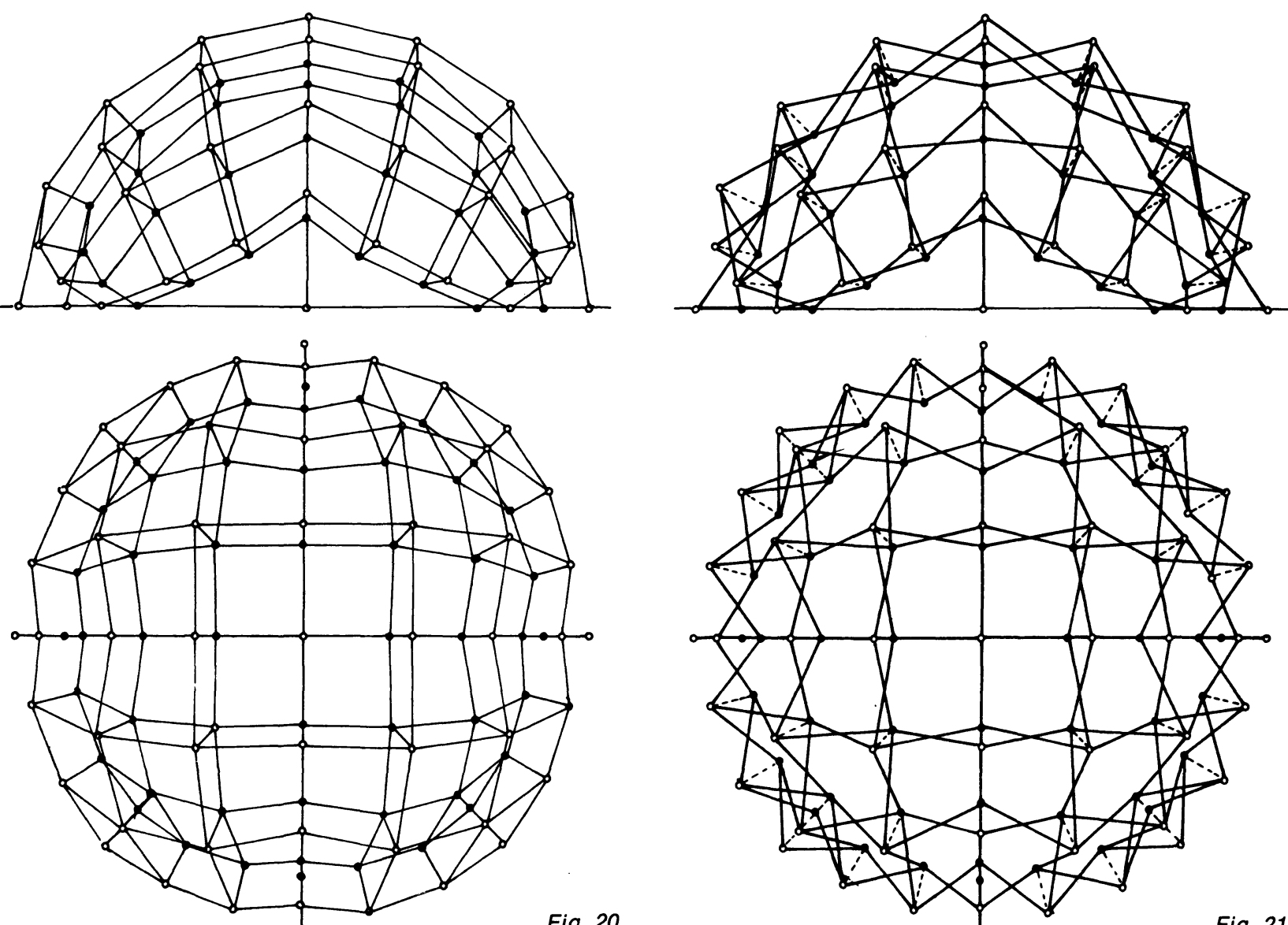

Fig. 20

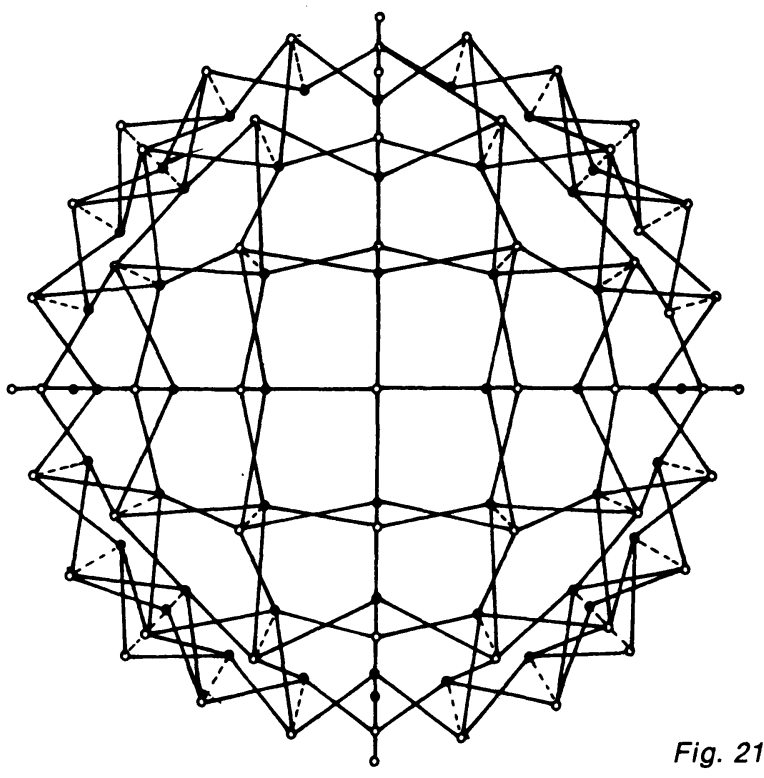

http://informesdelaconstruccion.revistas.csic.es 

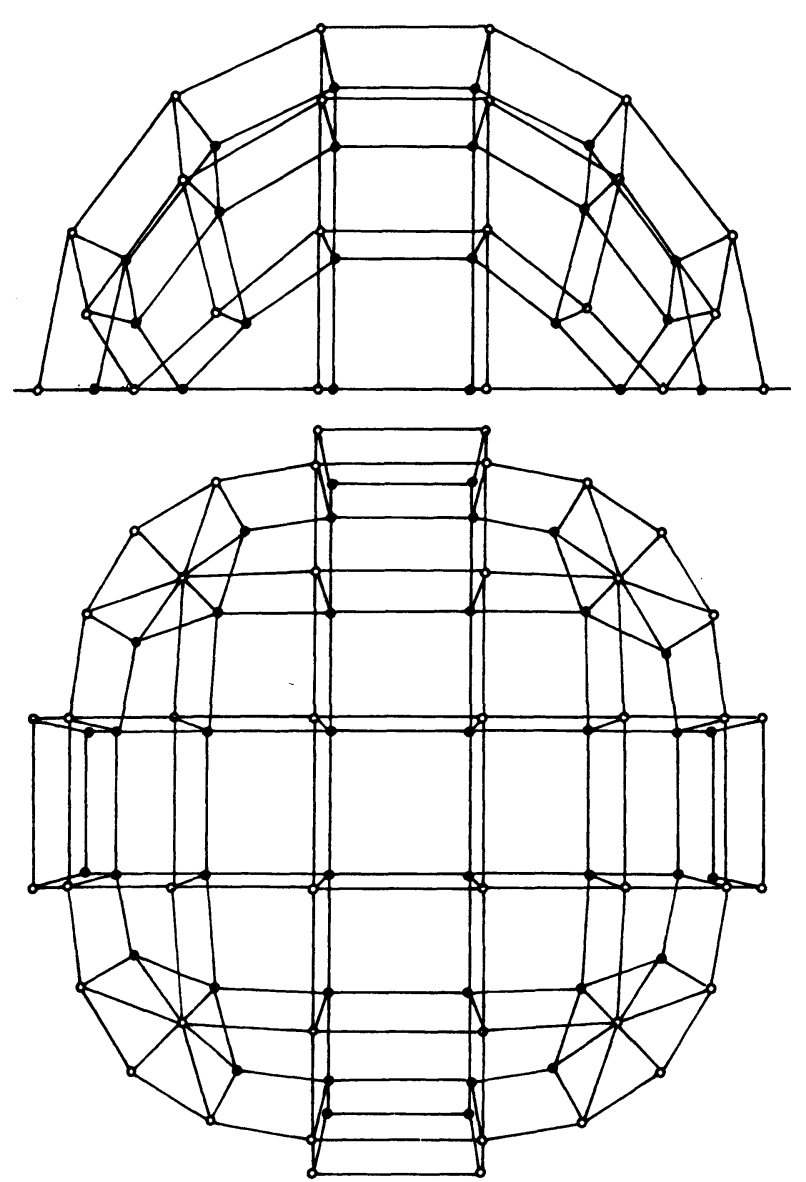

Fig. 22
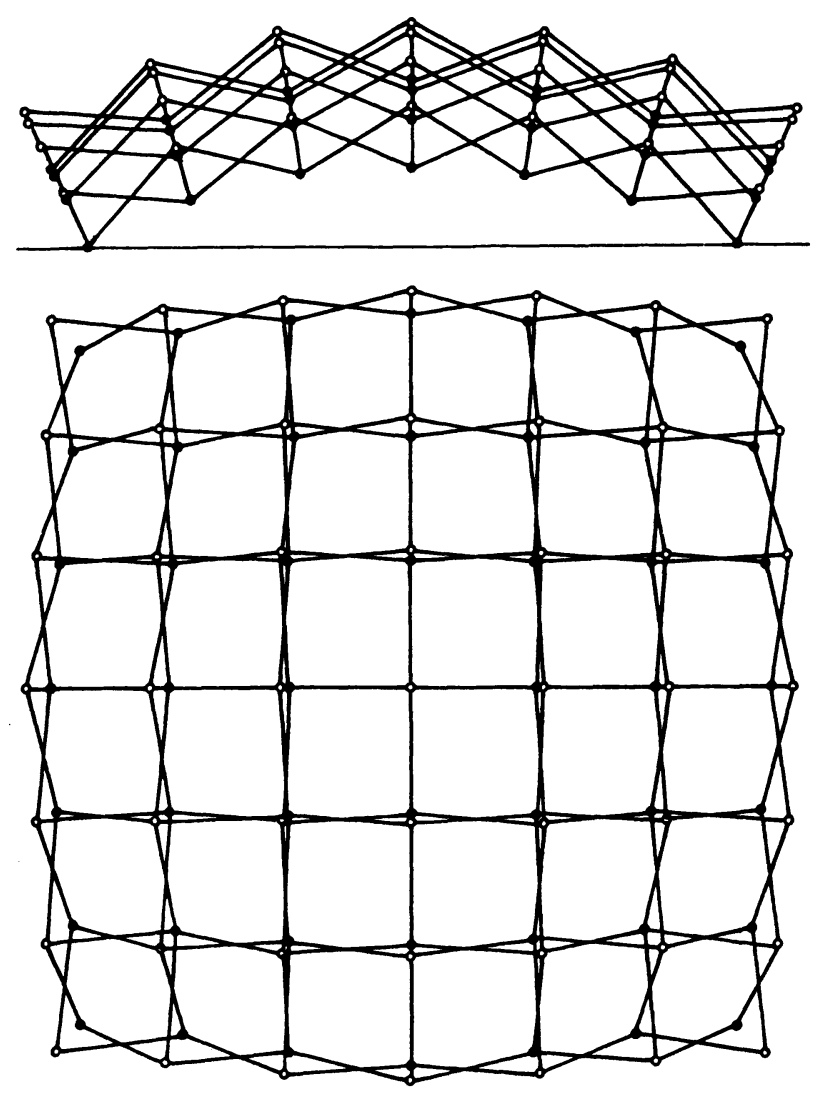

Fig. 24
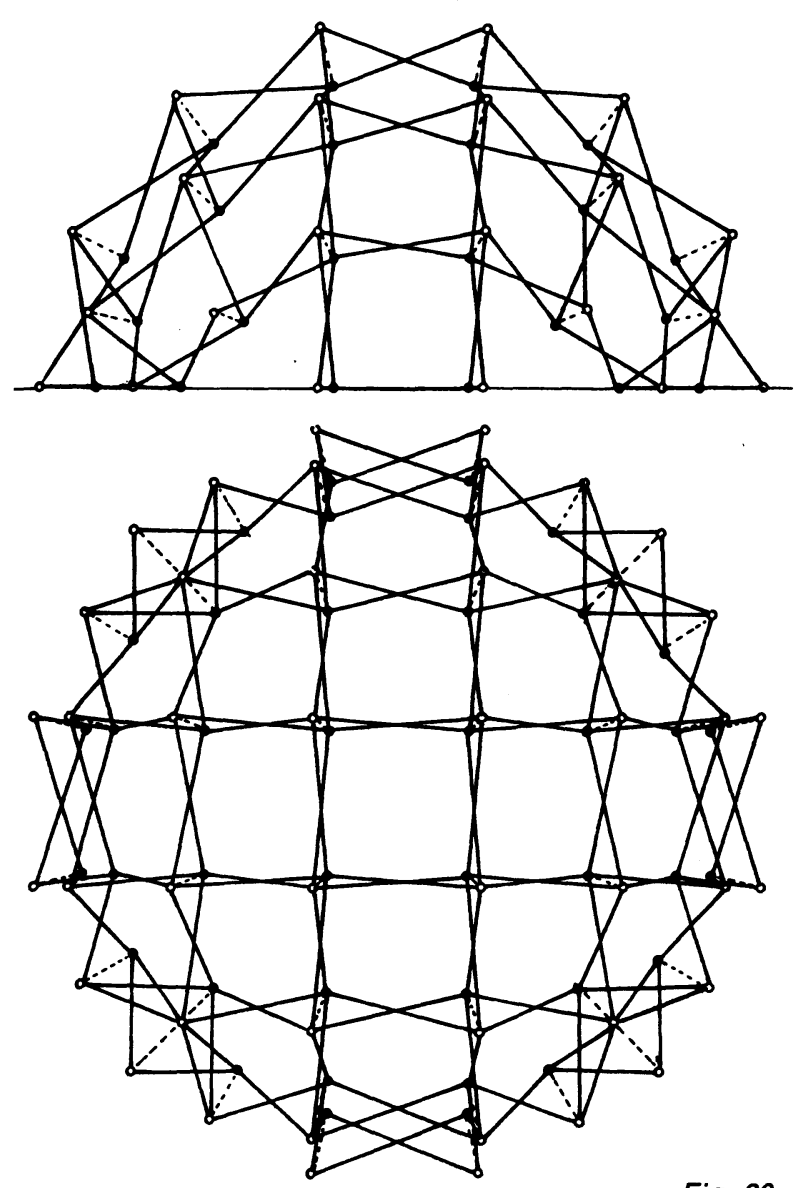

Fig. 23

Esta malla es inestable puesto que puede sufrir distorsiones angulares. Pero este efecto puede evitarse triangulando algunos elementos como se hizo en el caso de las mallas cilíndricas.

Las Fig. 21 y 23 muestran las dos posibilidades básicas para resolver un casquete con barras de igual longitud. Con barras de distinta longitud existen otras posibilidades. En la Fig. 24 se ve una configuración obtenida por proyección como se hizo en la Fig. 7 . 


\section{MALLAS ESFERICAS TRIANGULARES}

En la Fig. 13 podemos curvar las tres familias A, B y $C$ para obtener una malla esférica. Por supuesto esto tiene grandes dificultades, puesto que la geometría básica es muy rigida y da lugar a barras de distintas longitudes.

Para obtener éstas con una retícula esférica determinada, utilizamos las condiciones de compatibilidad inicial y final, es decir, garantizamos la geometría en la posición plegada y completamente desplegada. Pero estas condiciones, que son necesarias, no son suficientes puesto que la estructura pasa por estados intermedios incompatibles que precisan forzarla, aportando energía. A partir del punto en que alcanzamos la máxima incompatibilidad, la estructura devuelve la energía almacenada plegándose o desplegándose y estabilizán-
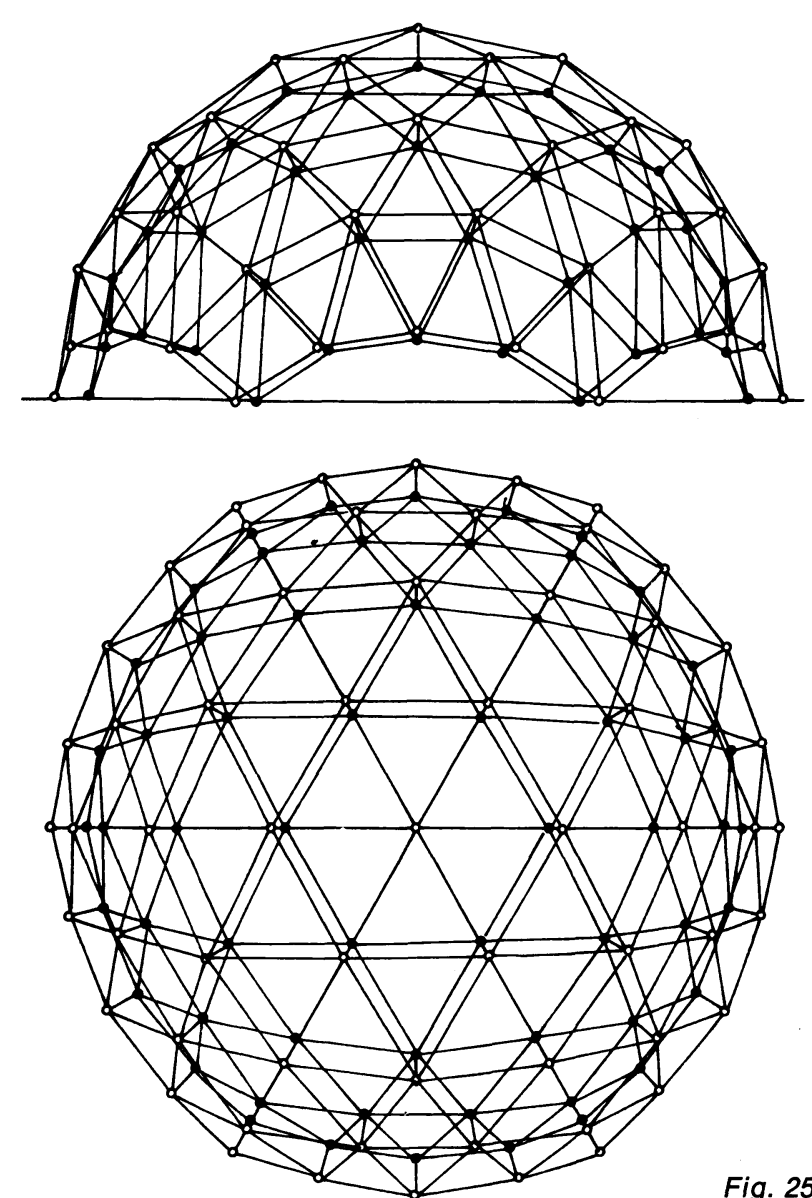

Fig. 25 dose en una de las posiciones límite, las únicas de diseño compatible. Si las barras no son demasiado rígidas esto puede ser una ventaja porque obtenemos estructuras que se autoestabilizan.

El ajuste de una malla triangular sobre una esfera puede hacerse de diversos modos, pero básicamente proyectanto sobre una superficie esférica una malla contenida en el plano ecuatorial, con origen en un foco situado sobre el eje polar de la esfera (Figs. 26 y 27). Según la posición de la malla obtendremos estructuras como las de las Figs. 27 ó 28. Es conveniente elegir el foco de modo que las longitudes de las barras sean lo más parecidas posible.

La malla de la Fig. 30 se ha obtenido proyectando desde el centro de la esfera una malla contenida en una pirámide hexagonal.
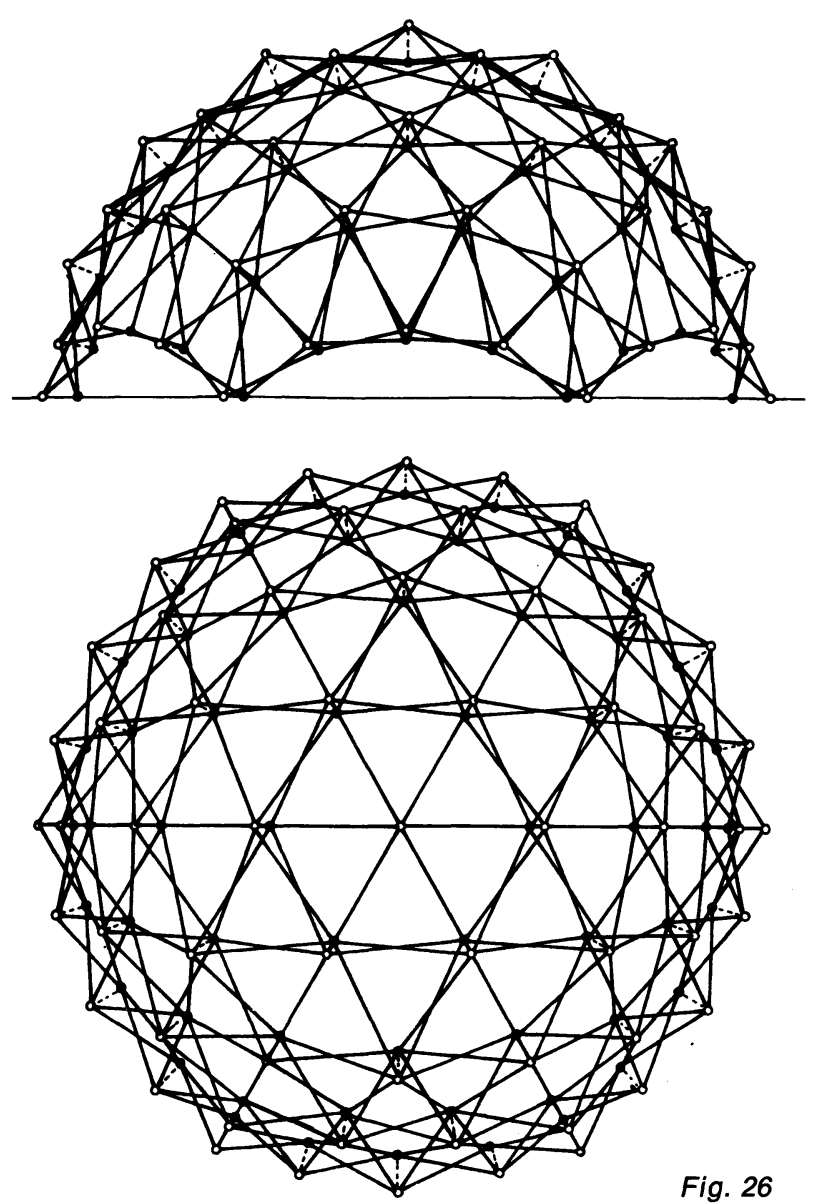

http://informesdelaconstruccion.revistas.csic.es 

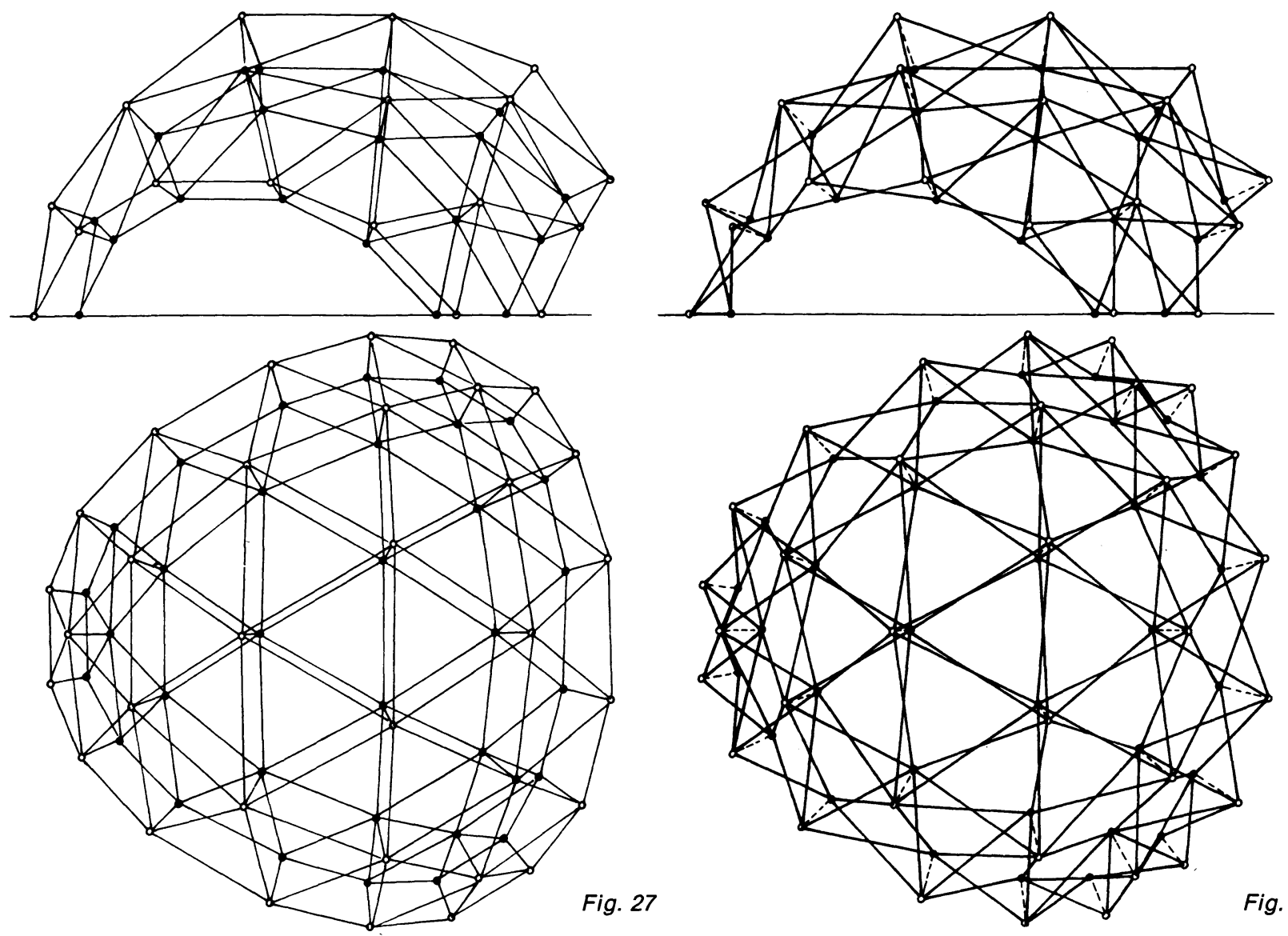

Fig. 28
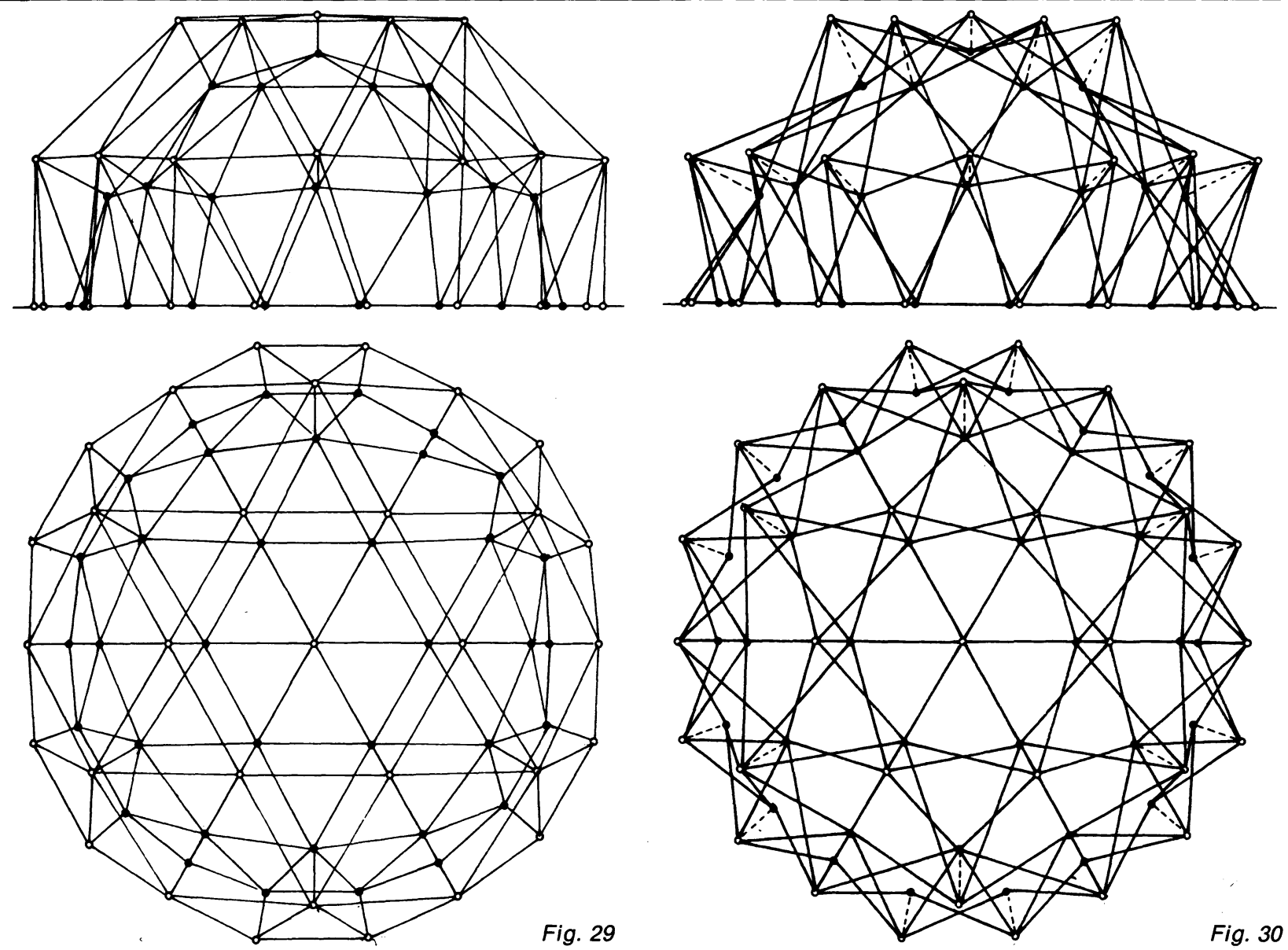

(c) Consejo Superior de Investigaciones Científicas Licencia Creative Commons 3.0 España (by-nc) 


\section{MALLAS GEODESICAS}

Para minimizar la distorsión en las mallas cuadrangulares o triangulares sobre una esfera podemos utilizar poliedros geodésicos.

Las mallas desplegables se obtienen, en este caso, sustituyendo cada uno de los lados del poliedro por aspas que satisfagan las condiciones de compatibilidad y con los nudos superiores e inferiores alineados con el centro de la esfera. Podemos utilizar poliedros basados en todo tipo de polígonos y cualquier orden de subdivisión.

Este tipo de mallas tiene los mismos problemas de compatibilidad en fases intermedias que vimos antes y es necesario el aporte de energía para el desplegado o el plegado que luego será devuelta para la estabilización del conjunto.
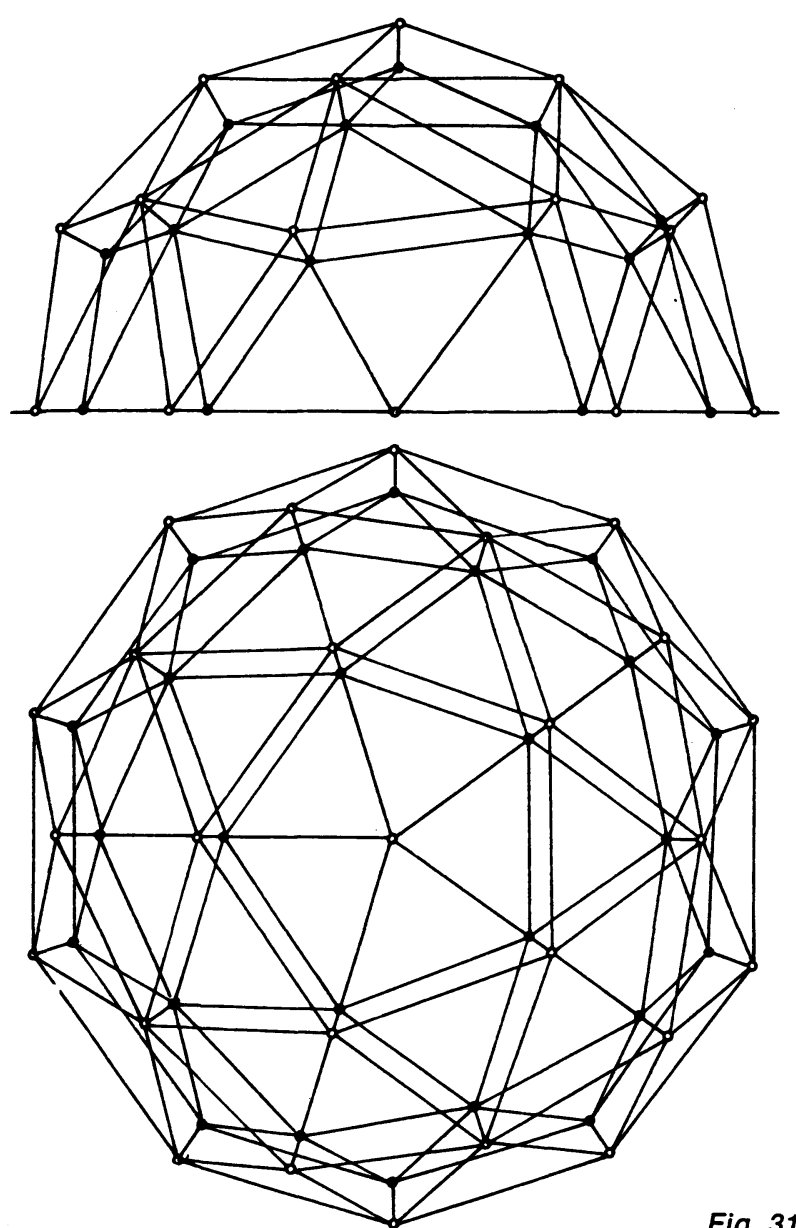

Fig. 31
Las frecuencias de subdivisión pequeñas permiten cumplir las condiciones de compatibilidad en los estados extremos, pero pasan por estados incompatibles. Las frecuencias de subdivisión mayores que tres no pueden cumplir todas las condiciones iniciales, pero evolucionan mejor en las intermedias.

La Fig. 32 muestra una malla desplegable a partir de un icosaedro de frecuencia dos y la Fig. 34 de frecuencia cuatro. Ziegler (Ref. 13) fue el primero en construir y patentar una estructura geodésica en icosaedro. Los problemas de incompatibilidad los resolvió mediante artificios que aumentaban los grados de libertad del conjunto.

Mediante poliedros geodésicos menos rígidos (Figuras 35 y 36) pueden evitarse algunos de estos problemas; pero la estabilidad de estas estructuras todavía no ha sido comprobada.
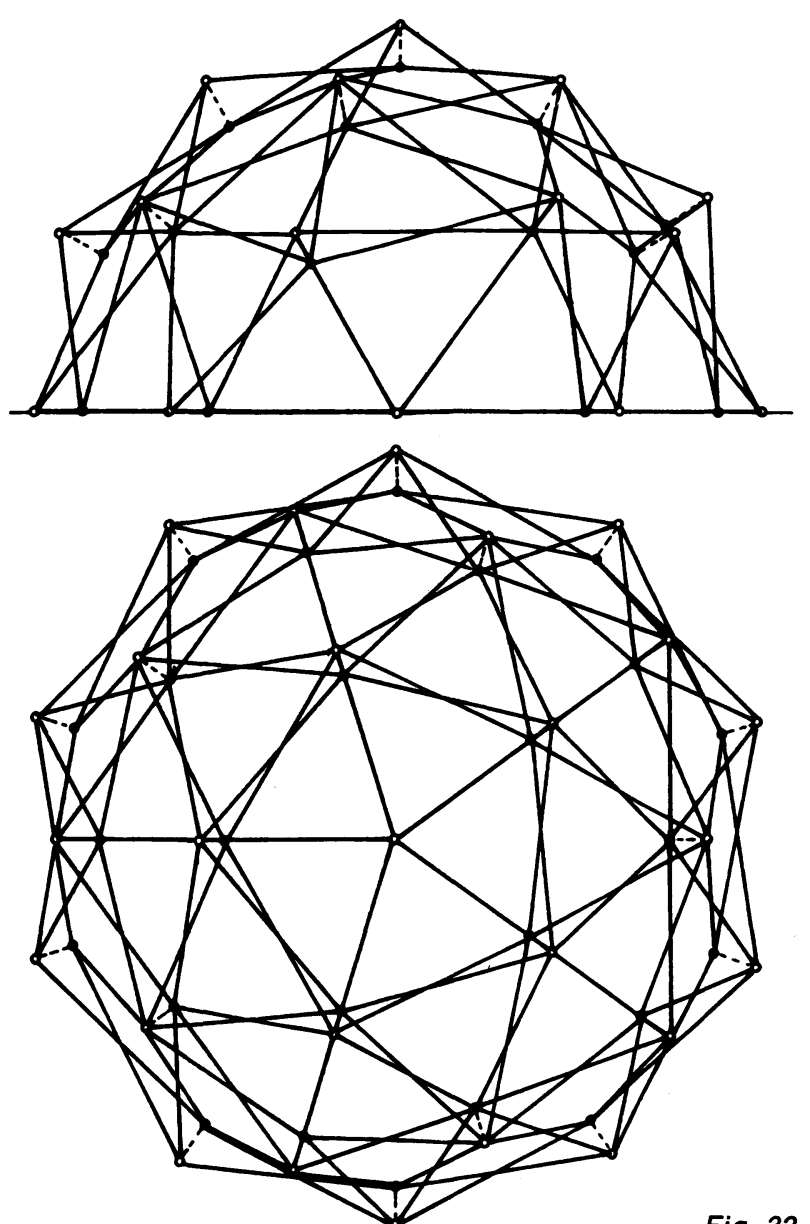

Fig. 32 

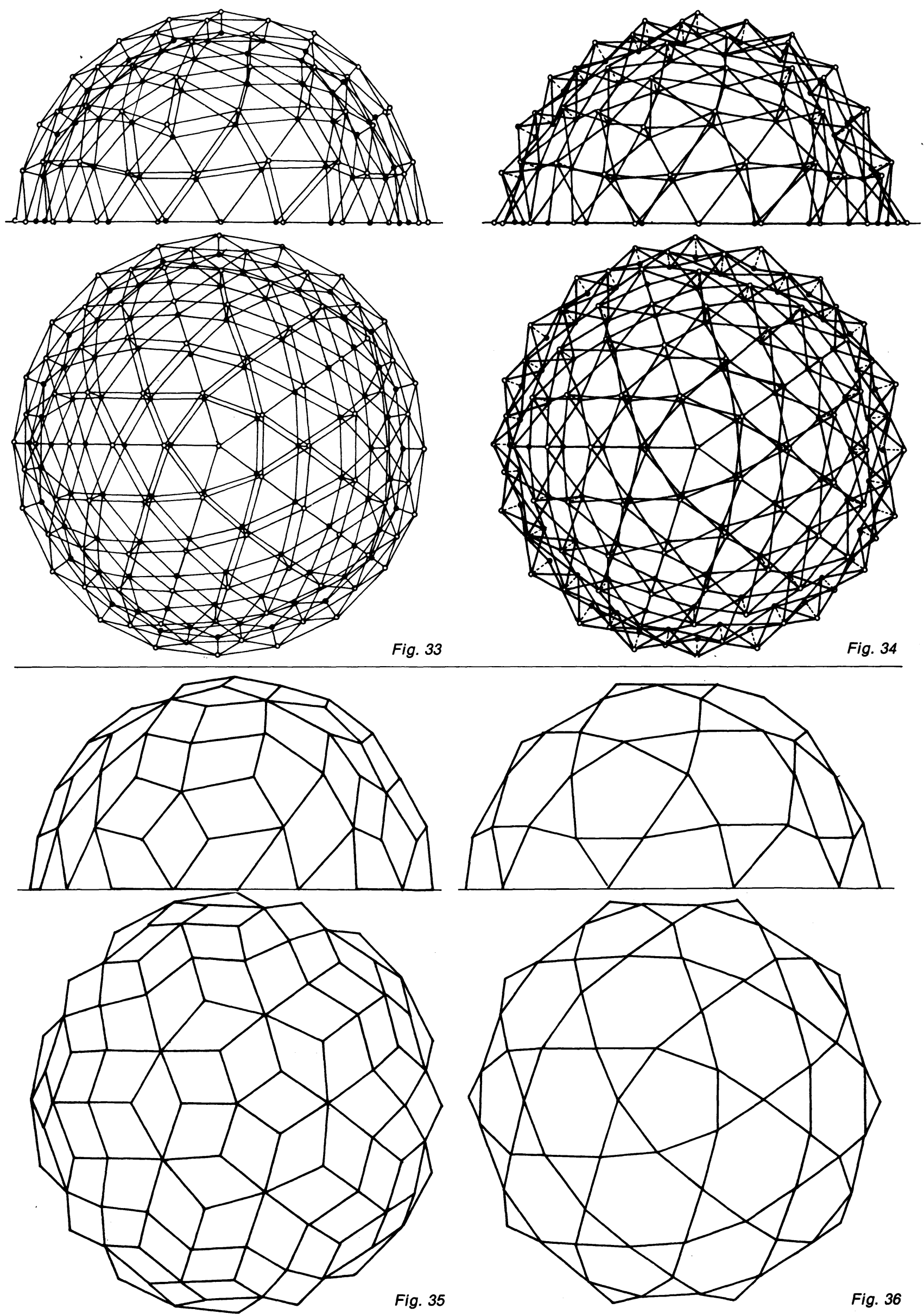

(c) Consejo Superior de Investigaciones Científicas 


\section{MALLAS ESFERICAS TIPO LAMELA}

Estas mallas se basan en un despliegue de rombos desde el polo de la esfera manteniendo constante el número de ellos en cada paralelo. El número de rombos que llega al polo define el número de orden de este tipo de estructuras (Fig. 38).

Estas estructuras son fáciles de diseñar, pueden resolverse con barras de la misma longitud y son compatibles en todo el proceso de apertura. Precisamente por ello son inestables y necesitan reforzarse con elementos adicionales para mantenerlas desplegadas.
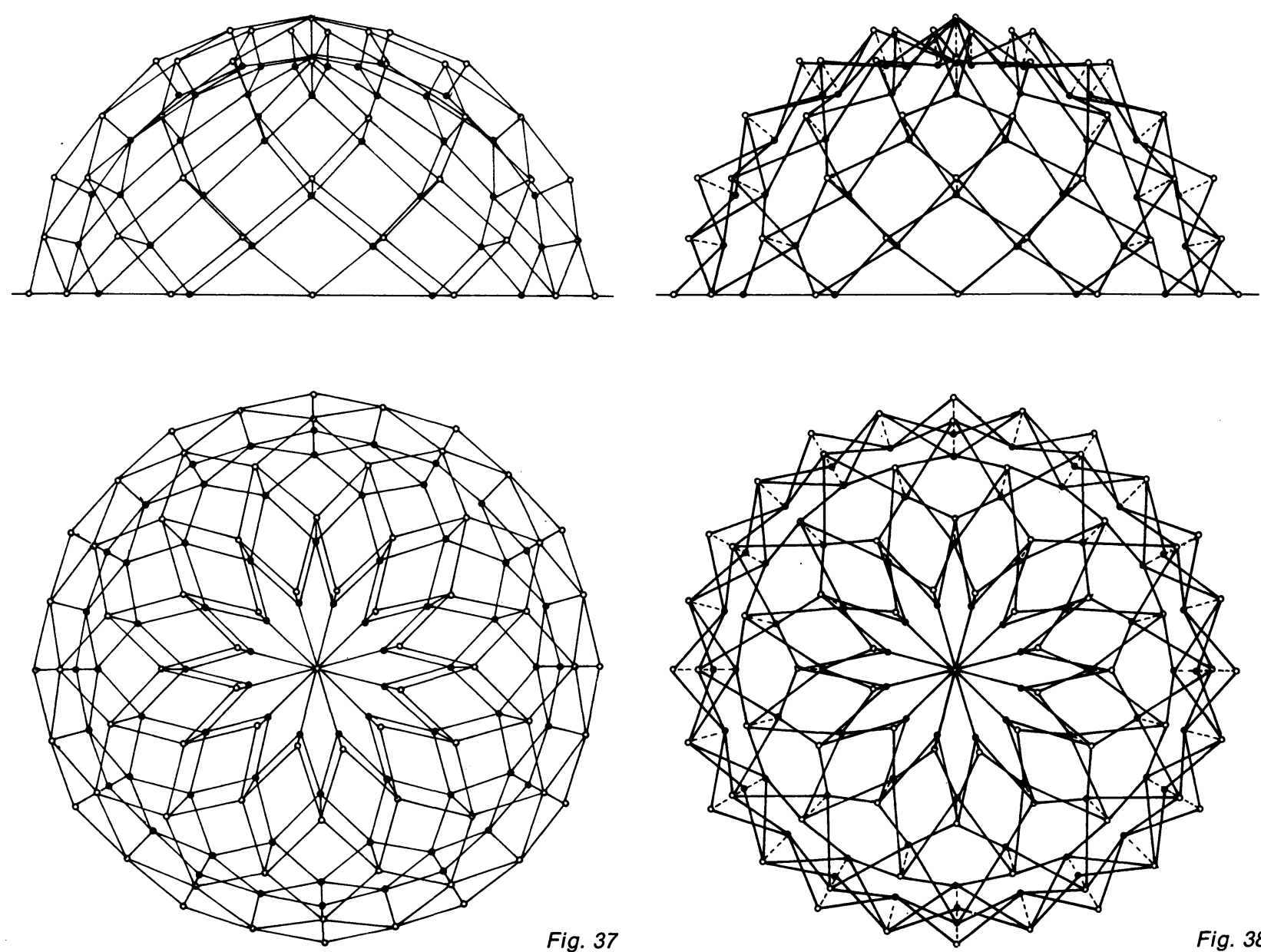

Fig. 37

Fig. 38 


\section{MALLAS ESFERICAS CON ASPAS TRIPLES}

E. P. Piñero fue el que más trabajó con este tipo de estructuras y fue el pionero en su utilización (Refs. 10 y 11). Se basan en elementos excéntricos como el de la Fig. 2. Aunque se generan desde retículas triangulares, los elementos tienen dos grados de libertad más que las aspas $y$, por tanto, los problemas de compatibilidad son menores, tanto que los conjuntos deben ser rigidizados adicionalmente.

Básicamente podemos obtener mallas curvas doblando mallas planas o ajustándolas a poliedros geodési$\mathrm{cos}$, de igual modo que en las estructuras de aspa ( $\mathrm{Fi}$ gura 40).

Una dificultad suplementaria es la resolución de encuentro triple del nudo intermedio de cada módulo.
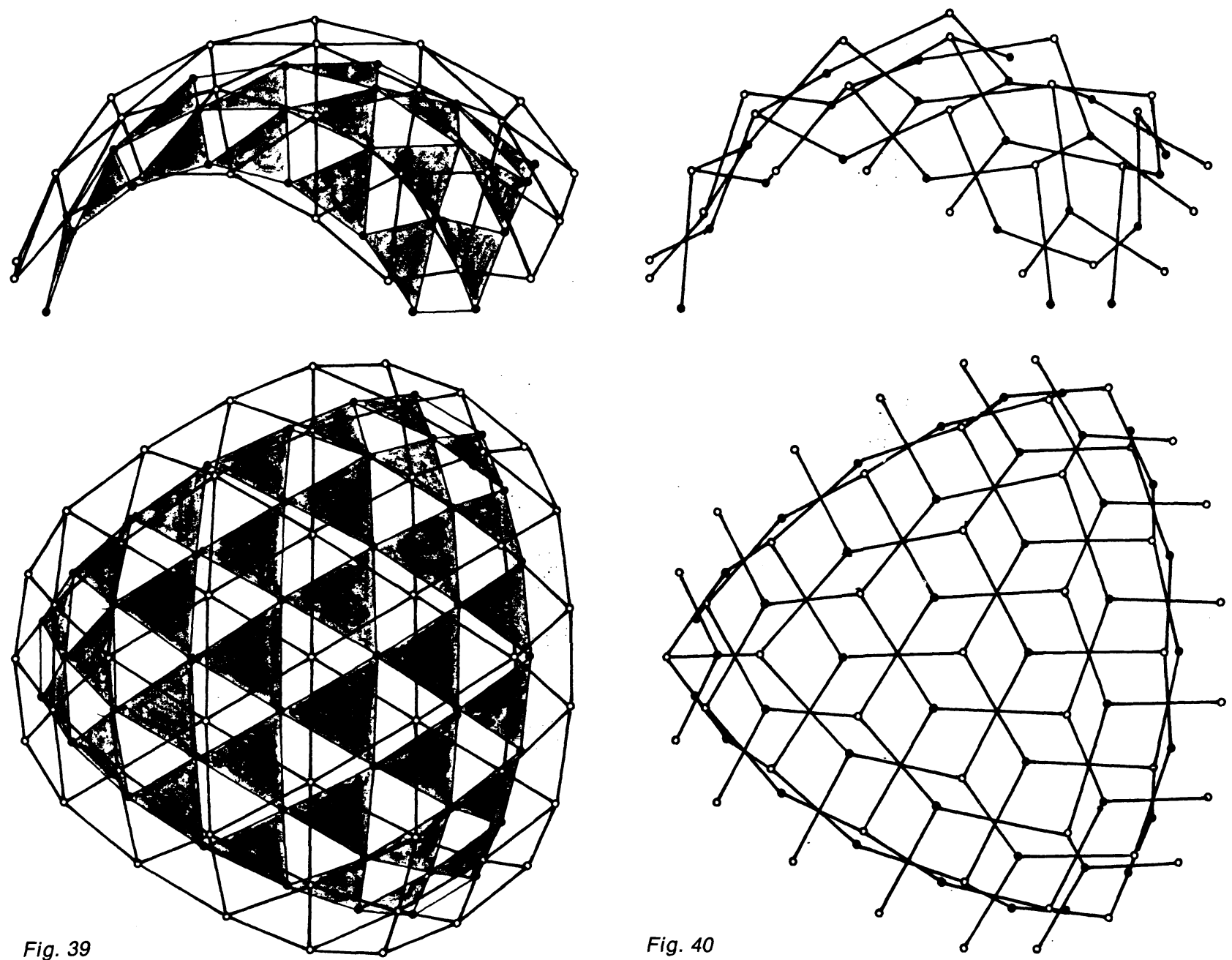


\section{OTROS TIPOS DE MALLA}

El catálogo anterior, evidentemente, permite muchas variables. Podemos citar las que tienen origen en elementos como el de la Fig. 3, que pueden adaptarse a mallas cilíndricas o esféricas.

Igualmente podemos citar otro tipo de estructuras como los de las Figs. 41 y 42 que son muy rígidas pero no se pliegan de un modo compacto.

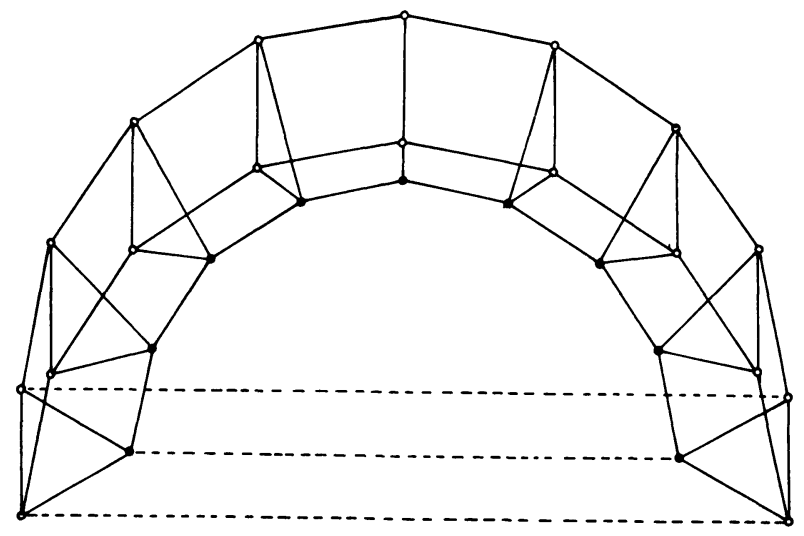

Fig. 41

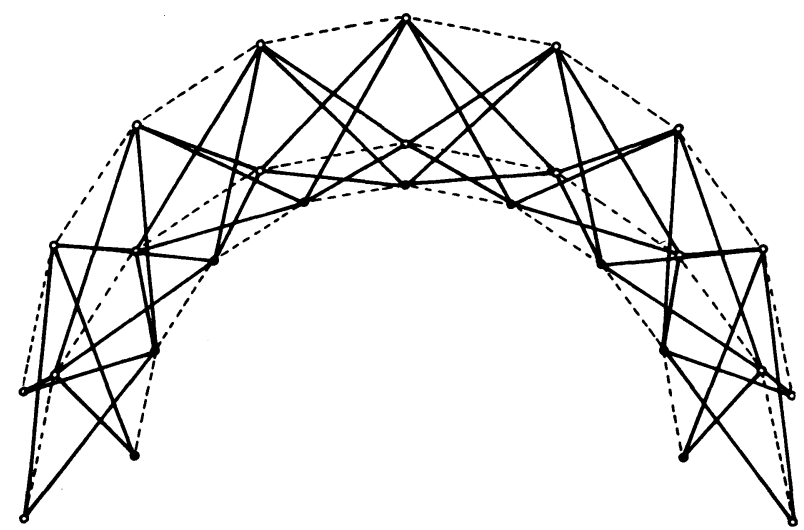

Fig. 42

\section{PLANTEAMIENTO DEL CALCULO}

La formulación matricial de las estructuras desplegables presenta ciertos problemas derivados del hecho de que, sus piezas constituyentes, son barras articuladas en sus extremos que tienen un apoyo central sobre otra pieza. Esto hace que los nudos centrales deban considerarse como articulados si estudiamos la interacción de una barra con otra y empotrados si estudiamos la barra como continua. Ninguno de los programas de ordenador habituales puede resolver este problema.

Sin embargo es posible plantear unas ecuaciones matriciales que expresen el equilibrio de esta barra y, en consecuencia, formular un nuevo programa capaz de calcular este tipo de estructuras.

Una barra caracteristica de estas estructuras se puede considerar articulada en sus extremos y apoyada en un punto interior (Fig. 43). La reacción exterior debida a la barra que se articula sobre ella puede descomponerse en tres fuerzas $X, Y Z$. De ellas la fuerza $X$, actuante sobre el eje de la pieza, modificará los valores de los axiles en los dos tramos de la barra y las fuerzas transversales $Y, Z$ provocarán flexiones sobre la barra. El equilibrio total se representa en la Fig. 44. Los cuatro esfuerzos $N_{1}, N_{2}, P_{1}$ y $P_{2}$ definen totalmente el equilibrio de la barra.

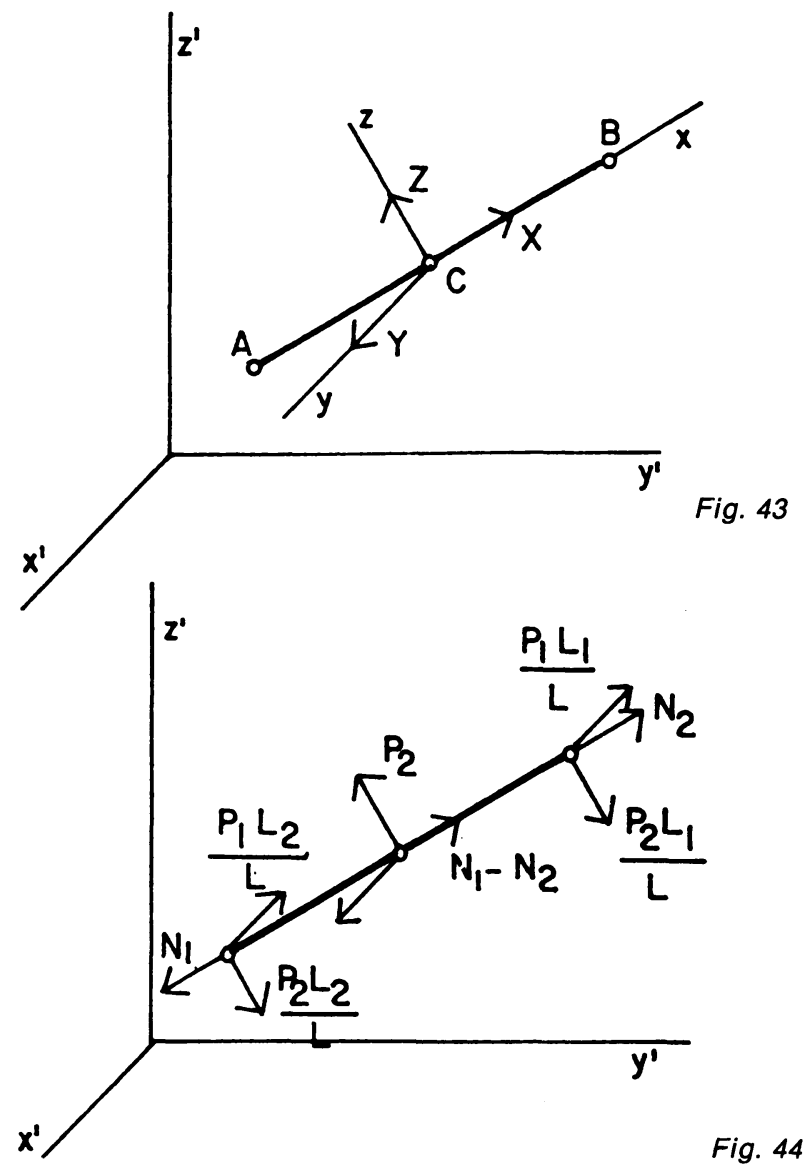


A efectos de esfuerzos axiles la respuesta de la barra será similar a la de una barra de una estructura de nudos articulados. En consecuencia, llamando $u_{1}$ al alargamiento del primer tramo de la barra y $u_{2}$ al del segundo (Figs. 45 y 46 )

$N_{1}=\frac{E \cdot A}{L_{1}} u_{1} \quad N_{2}=\frac{E \cdot A}{L_{2}} u_{2}$

A efectos de las fuerzas transversales en el nudo central, suponemos la barra articulada en sus extremos y sometida a una carga transversal sobre dicho nudo. En este caso

$v=\frac{1}{E \cdot I_{1}} \cdot \frac{P_{1} \cdot L_{1}^{2} \cdot L_{2}^{2}}{3 \cdot L}$

En consecuencia

$P_{1}=\frac{3 \cdot E \cdot I_{1} \cdot L}{L_{1}^{2} \cdot L_{2}^{2}} v \quad P_{2}=\frac{3 \cdot E \cdot I_{2} \cdot L}{L_{1}^{2} \cdot L_{2}^{2}} w$
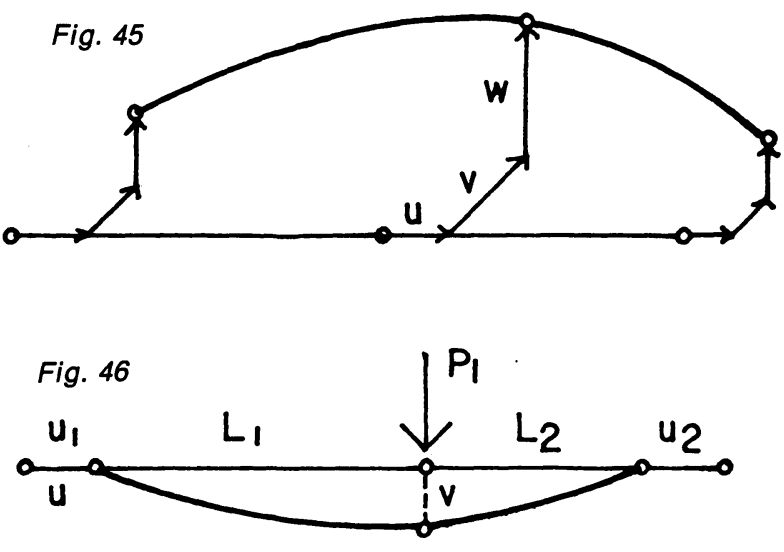

$Y$ en forma matricial obtenemos la matriz de rigidez en coordenadas locales.

$$
\left|\begin{array}{c}
N_{1} \\
N_{2} \\
P_{1} \\
P_{2}
\end{array}\right|=\left|\begin{array}{cccc}
\frac{E A}{L_{1}} & 0 & 0 & 0 \\
0 & \frac{E A}{L_{2}} & 0 & 0 \\
0 & 0 & \frac{3 E I_{1} L}{L_{1}^{2} L_{2}^{2}} & 0 \\
0 & 0 & 0 & \frac{3 E I_{2} L}{L_{1}^{2} L_{2}^{2}}
\end{array}\right|\left|\begin{array}{c}
u_{1} \\
u_{2} \\
v \\
v
\end{array}\right|
$$

(8)
Para definir la matriz de compatibilidad suponemos unos ejes locales tales que el eje $x$ lleva la dirección de la barra, el eje $y$ es horizontal y perpendicular al $x$ $y$ el eje $z$ perpendicular a los $x e y$. Este criterio es válido salvo para barras verticales, en cuyo caso los ejes $y, z$ son ambos horizontales. Para resolver la ambigüedad se toma como eje $z$ el eje horizontal que corta al eje vertical $z^{\prime}$ y como eje $y$ el perpendicular a los otros dos ejes. La ley de transformación entre los dos sistemas será:

$$
\left|\begin{array}{l}
x \\
y \\
z
\end{array}\right|=\left|\begin{array}{lll}
\cos \alpha_{1} & \cos \beta_{1} & \cos \gamma_{1} \\
\cos \alpha_{2} & \cos \beta_{2} & \cos \gamma_{2} \\
\cos \alpha_{3} & \cos \beta_{3} & \cos \gamma_{3}
\end{array}\right|\left|\begin{array}{l}
x^{\prime} \\
y^{\prime} \\
z^{\prime}
\end{array}\right|
$$

En donde las relaciones entre los dos sistemas nos permiten ponerla en la forma:
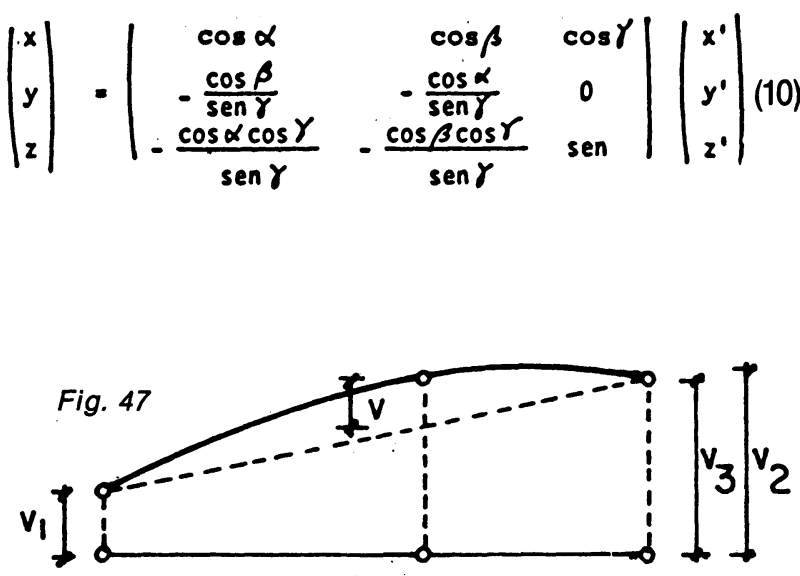

La matriz de compatibilidad se obtendrá poniendo los desplazamientos de la barra en función de los desplazamientos de los nudos (Fig. 47) 


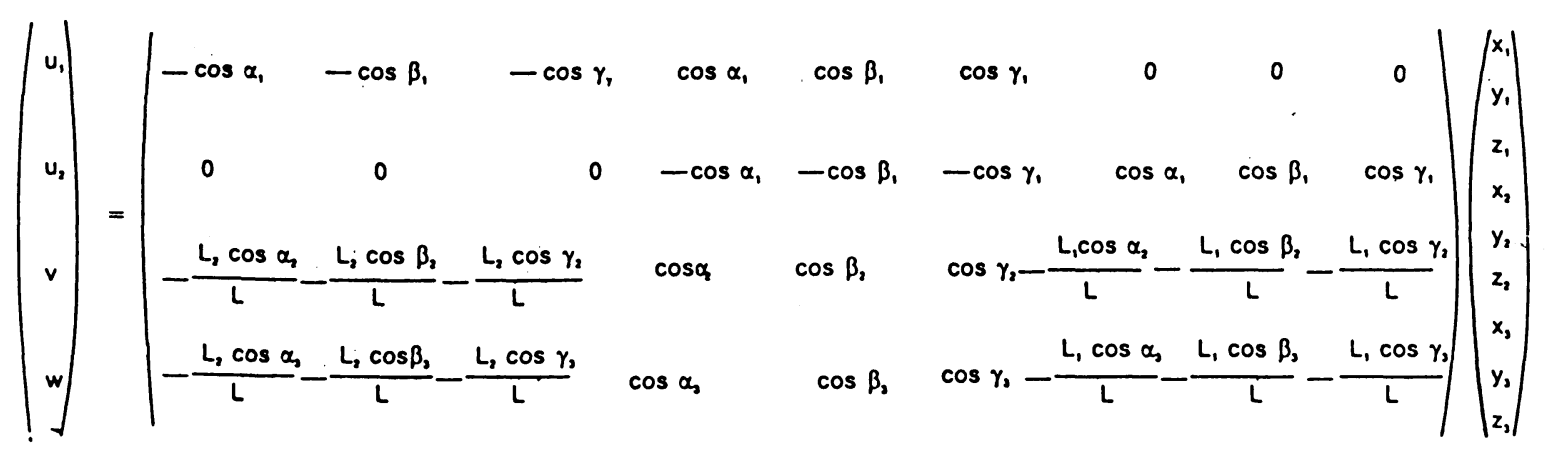

Planteando las ecuaciones anteriores en coordenadas globales tenemos:

Ecuación de rigidez

Ecuación de compatibilidad

Ecuación de equilibrio

En consecuencia

$$
\begin{aligned}
& <\mathrm{P}\rangle=\langle\mathrm{K}\rangle\langle\mathrm{Z}\rangle \\
& <\mathrm{Z}\rangle=\langle\mathrm{A}\rangle\langle\mathrm{X}\rangle \\
& \langle\mathrm{L}\rangle=\langle\mathrm{A}\rangle^{t} \\
& \langle\mathrm{~L}\rangle=\langle\mathrm{A}\rangle^{t}\langle\mathrm{~K}\rangle\langle\mathrm{A}\rangle\langle\mathrm{X}\rangle\langle\mathrm{S}\rangle\langle\mathrm{X}\rangle
\end{aligned}
$$

\begin{tabular}{|c|c|c|c|c|c|c|}
\hline $\begin{array}{l}a+m \cdot k_{1} \\
\text { sim. }\end{array}$ & $\begin{array}{ll}b+n \cdot k_{1} & c+o \cdot k_{1} \\
d+p \cdot k_{1} & e+q \cdot k_{1} \\
& f+r \cdot k_{1}\end{array}$ & $\begin{array}{c}-a-m \cdot k_{3} \\
\text { sim. }\end{array}$ & $\begin{array}{ll}-b-n \cdot k_{3} & -c \cdot-o \cdot k_{3} \\
-d-p \cdot k_{3} & -e \cdot q \cdot k_{3} \\
& -f-r \cdot k_{2}\end{array}$ & $\begin{array}{l}\mathrm{m} \cdot \mathrm{k}_{5} \\
\mathrm{sim} .\end{array}$ & $\begin{array}{l}n \cdot k_{5} \\
p \cdot k_{5}\end{array}$ & $\begin{array}{l}0 \cdot k_{5} \\
q \cdot k_{5} \\
r \cdot k_{5}\end{array}$ \\
\hline . & & $\begin{array}{c}a+g+m \\
\text { sim. }\end{array}$ & $\begin{array}{ll}b+h+n & c+i+o \\
d+j+p & e+k+q \\
& f+l+r\end{array}$ & $\begin{array}{l}-g-m \cdot k_{4} \\
\text { sim. }\end{array}$ & $\begin{array}{l}-h-n \cdot k_{4} \\
-j \cdot p \cdot k_{4}\end{array}$ & $\begin{array}{l}-i-o \cdot k_{4} \\
-k-q \cdot k_{4} \\
-l-r \cdot k_{4}\end{array}$ \\
\hline sim. & & sim. & & $\begin{array}{c}g+m \cdot k_{2} \\
\operatorname{sim} .\end{array}$ & $\begin{array}{l}h+n \cdot k_{2} \\
j+p \cdot k_{2}\end{array}$ & $\begin{array}{l}\mathrm{i}+\mathrm{o} \cdot \mathrm{k}_{2} \\
\mathrm{k}+\mathrm{q} \cdot \mathrm{k}_{2} \\
l+\mathrm{r} \cdot \mathrm{k}_{2}\end{array}$ \\
\hline
\end{tabular}

Por tanto la matriz de rigidez en coordenadas globales será:

siendo:

II $=\frac{3 E I_{1} L}{L_{1}^{2} L_{2}^{2}} \cos ^{2} \alpha_{2}+\frac{3 E I_{2} L}{L_{1}^{2} L_{2}^{2}} \cos ^{2} \alpha_{3}: n=\frac{3 E I_{1} L}{L_{1}^{2} L_{2}^{2}} \cos \alpha_{2} \cos \beta_{2}+\frac{3 E I_{2} L}{L_{1}^{2} L_{2}^{2}} \cos \alpha_{3} \cos \beta_{3}$

$i=\frac{E A}{L_{2}} \cos \alpha_{1} \cos \gamma_{1} ; j=\frac{E A}{L_{2}} \cos ^{2} \beta_{1}: k=\frac{E A}{L_{2}} \cos \beta_{1} \cos \gamma_{1} ; 1=\frac{E A}{L_{2}} \cos ^{2} \gamma_{1} \quad L_{1}=\frac{L_{2}^{2}}{L^{2}} ; k_{2}=\frac{L_{1}^{2}}{L^{2}} ; k_{3}=\frac{L_{2}}{L} ; k_{4}=\frac{L_{1}}{L} ; k_{5}=\frac{L_{1} L_{2}}{L^{2}}$

$0=\frac{3 E I_{1} L}{L_{1}^{2} L_{2}^{2}} \cos \alpha_{2} \cos \gamma_{2}+\frac{3 E I_{2} L}{L_{1}^{2} L_{2}^{2}} \cos \alpha_{3} \cos \gamma_{3} ; p=\frac{3 E I_{1} L}{L_{1}^{2} L_{2}^{2}} \cos ^{2} \beta_{2}+\frac{3 E I_{2} L}{L_{1}^{2} L_{2}^{2}} \cos ^{2} \beta_{3} \quad a=\frac{E A}{L_{1}} \cos ^{2} \alpha_{1} ; b=\frac{E A}{L_{1}} \cos \alpha_{1} \cos \beta_{1} ; c=\frac{E A}{L_{1}} \cos \alpha_{1} \cos \gamma_{1} ; d=\frac{E A}{L_{1}} \cos ^{2} \beta_{1}$ $a=\frac{3 E I_{1} L}{L_{1}^{2} L_{2}^{2}} \cos \beta_{2} \cos \gamma_{2}+\frac{3 E I_{2} L}{L_{1}^{2} L_{2}^{2}} \cos \beta_{3} \cos \gamma_{3} ; r=\frac{3 E I_{1} L}{L_{1}^{2} L_{2}^{2}} \cos ^{2} \gamma_{2}+\frac{3 E I_{2} L}{L_{1}^{2} L_{2}^{2}} \cos ^{2} \gamma_{3} \quad e=\frac{E A}{\Gamma_{1}} \cos \beta_{1}^{3} \cos \gamma_{1} ; f=\frac{E A}{L_{1}} \cos ^{2} \gamma_{1} ; g=\frac{E A}{L_{2}} \cos ^{2} \alpha_{1} ; h=\frac{E A}{L_{2}} \cos \alpha_{1} \cos \beta_{1}$ 

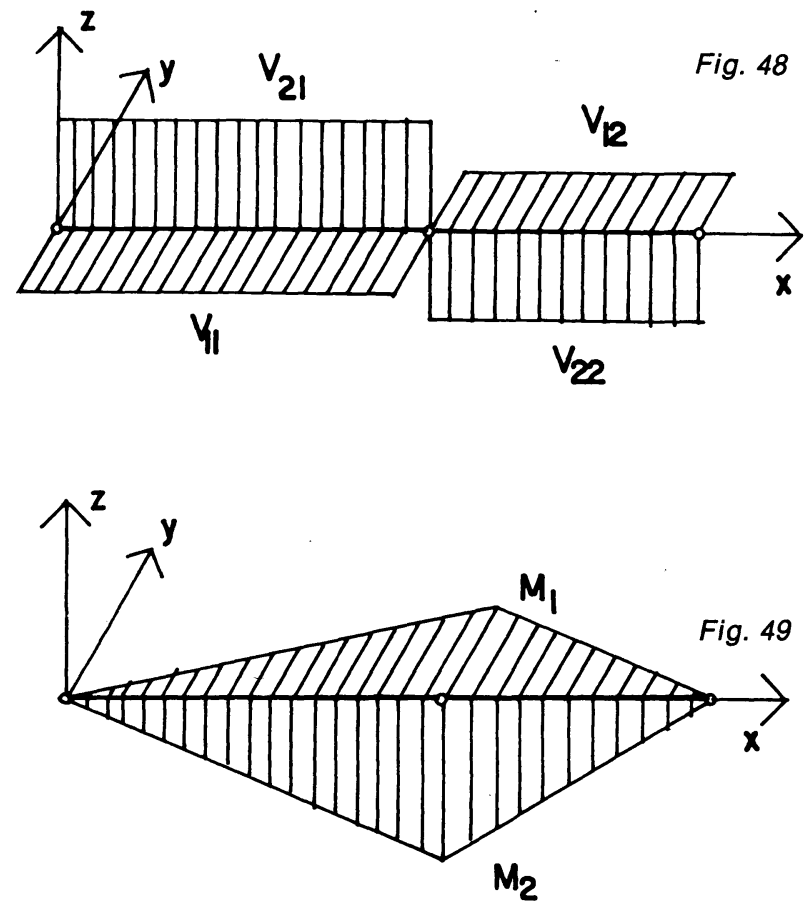

Para el cálculo de esfuerzos utilizaremos las ecuaciones (5) y (7) a partir de los desplazamientos que obtengamos de las ecuaciones (12). Generalmente es de mayor interés calcular los esfuerzos cortantes (Fig. 48) y los momentos flectores (Fig. 49) que las propias fuerzas transversales

$$
\begin{array}{ll}
V_{11}=\frac{P_{1} \cdot L_{2}}{L} & V_{12}=\frac{P_{1} \cdot L_{2}}{L} \\
V_{21}=\frac{P_{2} \cdot L_{2}}{L} & V_{22}=\frac{P_{2} \cdot L_{1}}{L} \\
M_{1}=\frac{P_{1} \cdot L_{1} \cdot L_{2}}{L} & M_{2}=\frac{P_{2} \cdot L_{1} \cdot L_{2}}{L}
\end{array}
$$

Una vez resuelto el cálculo es preciso comprobar que todos los nudos están en equilibrio. Por tanto calcularemos las fuerzas que actúan, en cada nudo, en coordenadas globales

$$
\begin{aligned}
& x_{1}=-N_{1} \cos \alpha_{1}-\frac{P_{1} L_{2}}{L} \cos \alpha_{2}-\frac{P_{2} L_{2}}{L} \cos \alpha_{3} ; r_{1}=-N_{1} \cos \beta_{1}-\frac{P_{1} L_{2}}{L} \cos \beta_{2}-\frac{P_{2} L_{2}}{L} \cos \beta_{3} \\
& z_{1}=-N_{1} \cos \gamma_{1}-\frac{P_{1} L_{2}}{L} \cos \gamma_{2}-\frac{P_{2} L_{2}}{L} \cos \gamma_{3} ; x_{2}=\left(N_{1}-N_{2}\right) \cos \alpha_{1}+P_{1} \cos \alpha_{2}+P_{2} \cos \alpha_{3} \\
& r_{2}=\left(N_{1}-N_{2}\right) \cos \beta_{1}+P_{1} \cos \beta_{2}+P_{2} \cos \gamma_{3}: z_{2}=\left(N_{1}-N_{2}\right) \cos \gamma_{1}+P_{1} \cos \gamma_{2}+P_{2} \cos \gamma_{3} \\
& x_{3}=N_{2} \cos \alpha_{1}-\frac{P_{1} L_{2}}{L} \cos \alpha_{2}-\frac{P_{2} L_{2}}{L} \cos \alpha_{3}: r_{3}=N_{2} \cos \beta_{1}-\frac{P_{1} L_{2}}{L} \cos \beta_{2}-\frac{P_{2} L_{2}}{L} \cos \beta_{3} \\
& z_{3}=N_{2} \cos \gamma_{1}-\frac{P_{1} L_{2}}{L} \cos \gamma_{2}-\frac{P_{2} L_{2}}{L} \cos \gamma_{3}
\end{aligned}
$$

Si las fuerzas totales en cada nudo están en equilibrio con las fuerzas exteriores, los cálculos son correctos.

En general, estas estructuras presentan un número relativamente importante de nudos y un ancho de banda considerable por las dificultades de su numeración. En consecuencia es importante tratar de reducir el número de elementos de las mismas, por aplicación de condiciones de simetría cuando ello sea posible.

En muchos casos la estructura tiene una simetria respecto a planos verticales que pasan por el eje $z$. En estas condiciones es preciso plantear la condición de que los desplazamientos se produzcan según un plano vertical que forma un cierto ángulo con los ejes globales (Figs. 50 y 51). La condición será que la componente del desplazamiento ortogonal al plano de simetría sea nula

$\delta_{\mathrm{X}} \operatorname{sen} \alpha-\delta_{\mathrm{Y}} \cos \alpha=0$

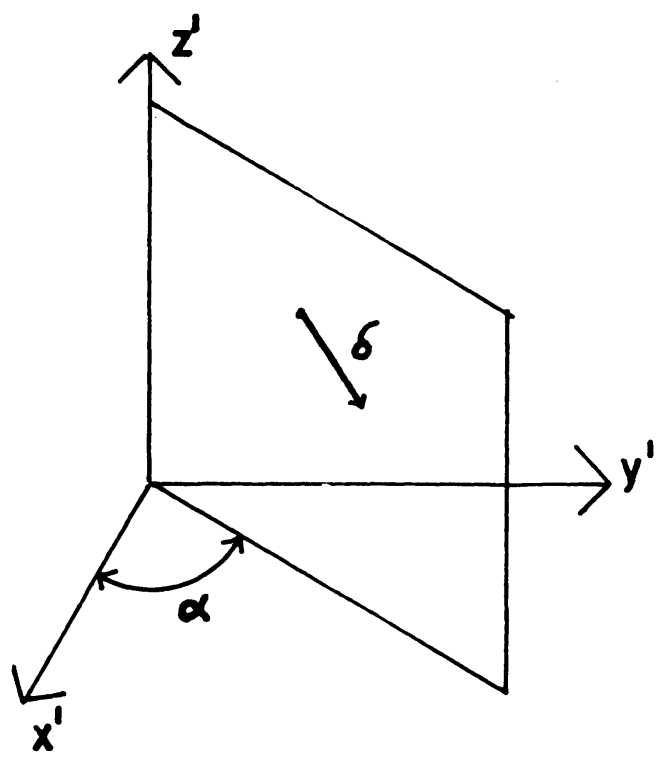

Fig. 50

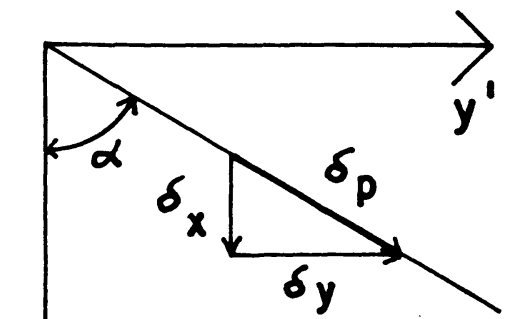

Fig. 51 
Al imponer esta condición, la matriz de rigidez en coordenadas globales que, referida a estos desplazamientos, tiene la forma:
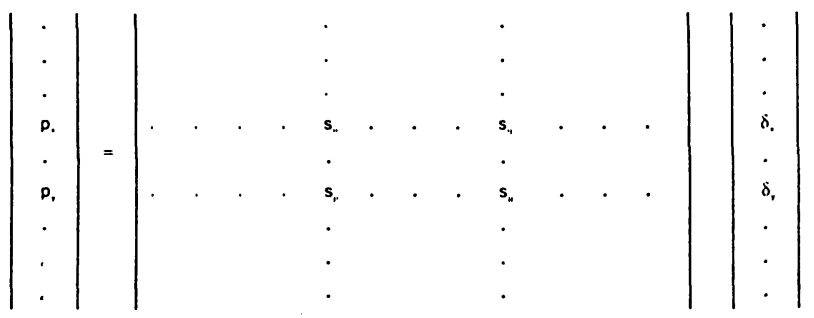

(16)

se tranforma en

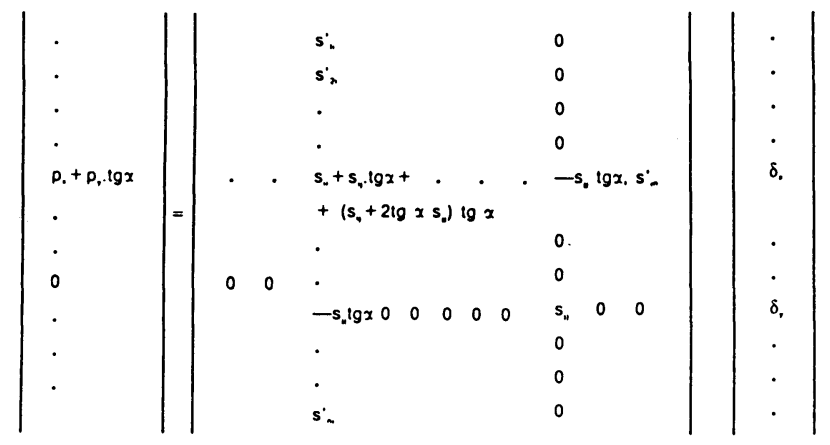

(17)

siendo

$s^{\prime}{ }_{n i}=s_{n i}+s_{n j} \operatorname{tg} \alpha$

$s_{\text {in }}^{\prime}=s_{\text {in }}+s_{\text {in }} \operatorname{tg} \alpha$

Este tipo de estructuras presenta ciertos problemas en cuanto a sus flechas, que pueden alcanzar grandes valores. Por otra parte su utilización arquitectónica exige un recubrimiento, generalmente textil, que pueda plegarse con las barras de la estructura y que quede tenso tras el desplegado. La resistencia a tracción de esta lámina textil mejora el comportamiento resistente del conjunto y disminuye las flechas. En cualquier caso habrá que considerar en el cálculo la existencia de unos elementos adicionales, cables o telas. Esta consideración introduce una dificultad doble. Es preciso formar y ensamblar las matrices de rigidez de los cables o telas y, además, hay que contemplar la posibilidad de que sólo pueden trabajar en tracción.

En este momento vamos a trabajar sólo con elementos lineales, y las telas serán tratadas como tales. Para resolver el primer problema utilizaremos la matriz de rigidez de la barra de una malla espacial con extremos articulados y la ensamblaremos con las ecuaciones anteriores. El axil de la barra se obtendrá a partir de los corrimientos de los extremos deducidos del cálculo.

(c) Consejo Superior de Investigaciones Científicas

Licencia Creative Commons 3.0 España (by-nc)
Para resolver el segundo problema procederemos, por iteraciones, recalculando la estructura después de suprimir los cables que trabajen en compresión. Generalmente basta una iteración para eliminar todos los cables comprimidos.

A partir de un programa elaborado como se ha descrito se han analizado varios ejemplos de mallas curvas, citados anteriormente, con los resultados genéricos siguientes:

- En mallas cilíndricas cuadradas se han analizado los tipos de la Fig. 52. Para rigidizar angularmente se situaron cables diagonales que reproducen el comportamiento de la cubierta textil. La primera experiencia es que los cables trabajan todos a compresión $y$, por tanto, son innecesarios salvo como rigidizadores. El caso (a) funciona bien y dio una flecha máxima de $9,6 \mathrm{~cm}$. El caso (b) dio, con $F=0,5 \mathrm{t}$, una flecha máxima de $24 \mathrm{~cm}$ y un comportamiento peor en todos los puntos. El caso (c) dio peores resultados que los anteriores.

- En mallas cilíndricas triangulares, con las mismas dimensiones y cargas, el comportamiento fue bueno y la flecha máxima de $9,7 \mathrm{~cm}$.

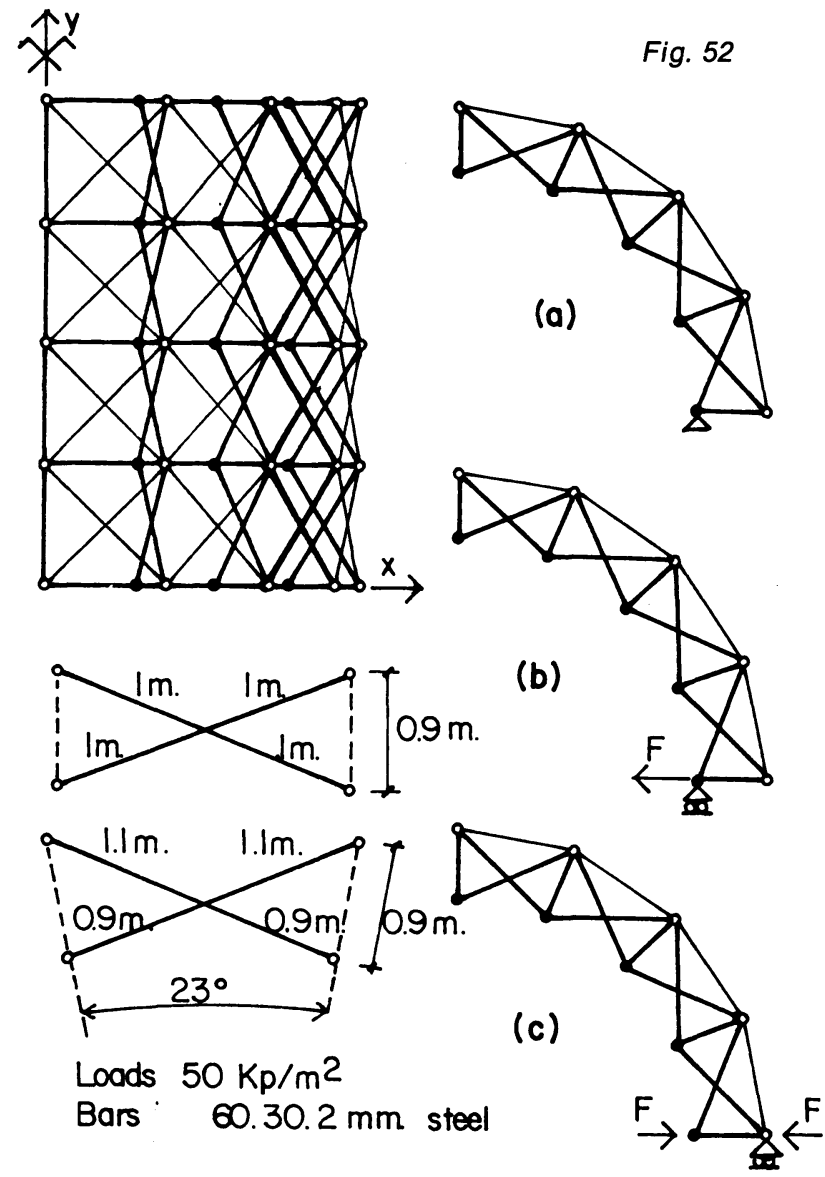

http://informesdelaconstruccion.revistas.csic.es 
No obstante, un análisis estructural más completo de estos casos y de los restantes, cuyo diseño se ha expuesto, está ahora mismo en proceso de investigación. Igualmente estamos realizando un análisis de segundo orden que incluye:

1. Efectos de los cambios de geometría y grandes desplazamientos.

2. Pandeo de las barras.

\section{REFERENCIAS}

(1) CLARKE, R. C. "The kinematics of a novel deployable space structural system". Third Int. Conf. on Space Structures Proc. Surrey 1984. Elsevier. pp. 820-822.

(2) ESCRIG, F. Patente española: "Sistema modular para la construcción de estructuras espaciales desplegables de barras". Num. 532117. Mayo 1984.

(3) ESCRIG, F. "Expandable Space Frame Structures". Third Int. Conf. on Space Structures Proc. Surrey 1984. Elsevier. pp. 845-850.

(4) ESCRIG. F. "Estructuras Espaciales de barras desplegables". Informes de la Construcción. Madrid. IET. Vol. 36. Num. 365. pp. 35-46.
(5) ESCRIG. F. "Expandable Space Structures". Space Structures Int. Journal. Vol 1. Num. 2. Elsevier. pp. 79-91.

(6) ESCRIG. F.; P. VALCARCEL, J. "Introducción a la geometría de las estructuras espaciales desplegables de barras". Boletín académico de la ETSA de La Coruña. Num. 3, Feb. 1986. pp. 48-57.

(7) ESCRIG. F.; P. VALCARCEL, J. "Analysis of Expandable Space Bar Structures". Int. Symp. on Membrane Structures and Space Frames Proc. IASS. Osaka. 1986. 8 pp.

(8) ESCRIG, F.; P. VALCARCEL, J. "Great Size Umbrellas solved with Expandable Bar Structures". First Int. Conf. on Lightweight Structures in Architecture. Sydney 1986. pp. $676-681$.

(9) MCNULTY, O. "Foldable Space Structures". First Int. Conf. on Lightweight Structures in Architecture". Sydney 1986. pp. 682-689.

(10) P. PIÑERO, E. "Materia-Estructura-Forma" Hogar y Arquitectura. Num. 40. Madrid 1962. pp. 25-30.

(11) P. PIÑERO, E. "Estructures reticulées". L'Architecture d'aujourd'hui, Vol. 141. Dic. 1968. pp. 76-81.

(12) P. VALCARCEL, J.; ESCRIG, F. "Bases del cálculo no lineal de estructuras desplegables espaciales" Bol. AC. de la ETSA de La Coruña, num. 7. Nov. 1987. 5 pp.

(13) ZEIGLER, T. U.S. Patent 4.026.313. May 1977.

\section{publicación del i.e.t.c.c.}

\section{ACUEDUCTOS ROMANOS EN ESPAÑA Carlos Fernández Casado Prof. Dr. Ing. de Caminos, Canales y Puertos}

Esta publicación se compone de una serie de artículos, publicados en la Revista "Informes de la Construcción", en los cuales se hace un análisis de los acueductos romanos que existen en España y el balance de las condiciones de conservación en que se encuentra cada uno de ellos, incluyendo referencias históricas y literarias. Se ha ilustrado con la reproducción de la valiosa documentación gráfica que posee el prestigioso autor.

Un volumen encuadernado en couché, a dos colores, de $21 \times 27$ centímetros, compuesto de 238 páginas, numerosos grabados, dibujos, fotos en blanco y negro y figuras de linea.

Precio: España, 1.500 ptas., 21 \$ USA.

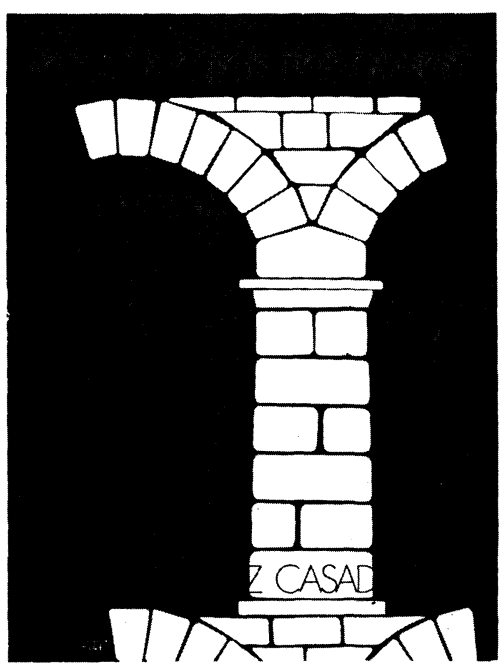

